\title{
Bringing Greater Accuracy to Europe's Healthcare Systems: The Unexploited Potential of Biomarker Testing in Oncology
}

\author{
Denis Horgan ${ }^{a} \quad$ Gennaro Ciliberto $^{b} \quad$ Pierfranco Conte $^{c} \quad$ David Baldwin $^{d}$ \\ Luis Seijo ${ }^{\text {Luis M. Montuenga }}{ }^{f}$ Luis Paz-Ares ${ }^{g}$ Marina Garassino ${ }^{h}$ \\ Frederique Penault-Llorca ${ }^{i}$ Fabrizia Galli ${ }^{j}$ Isabelle Ray-Coquard ${ }^{k}$ \\ Denis Querleu' Ettore Capoluongo ${ }^{m}$ Susana Banerjee ${ }^{\mathrm{n}} \quad$ Peter Riegman $^{\circ}$ \\ Keith Kerr ${ }^{p}$ Benjamin Horbach ${ }^{q}$ Reinhard Büttner ${ }^{r}$ Hein Van Poppel ${ }^{s}$ \\ Anders Bjartell ${ }^{t}$ Giovanni Codacci-Pisanelli ${ }^{u}$ Benedikt Westphalen ${ }^{v}$
} Fabien Calvo w Jasmina Koeva-Balabanova $^{x}$ Stephen Hally Angelo Paradiso ${ }^{z}$ Dipak Kalra $^{A}$ Christa Cobbaert ${ }^{B}$ Rocio Varea Menendez ${ }^{C}$ Zorana Maravic ${ }^{D}$ Vassiliki Fotaki ${ }^{D}$ Jaafar Bennouna ${ }^{E}$ Estelle Cauchin ${ }^{\mathrm{E}}$ Nuria Malats ${ }^{\mathrm{F}}$ Iñaki Gutiérrez-Ibarluzea ${ }^{G, H}$ Benjamin Gannon' Ken Mastris ${ }^{J}$ Chiara Berninia William Gallagher $^{\mathrm{K}}$ Simonetta Buglioni ${ }^{\mathrm{b}}$ Alastair Kent ${ }^{\mathrm{L}} \quad$ Elisabetta Munzone $^{\mathrm{M}}$ Ivica Belina ${ }^{N}$ Jan Van Meerbeeck ${ }^{O}$ Michael Duffy ${ }^{K}$ Elżbieta Sarnowska ${ }^{P}$ Beata Jagielska $^{\mathrm{P}}$ Sarah $\mathrm{Mee}^{\mathrm{Q}}$ Giuseppe Curigliano ${ }^{\mathrm{M}}$

\footnotetext{
${ }^{a}$ European Alliance for Personalised Medicine, Brussels, Belgium; ${ }^{b}$ IRCCS Istituto Nazionale

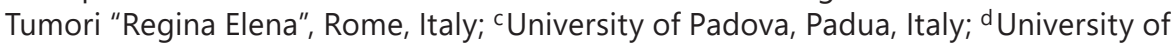
Nottingham, Nottingham, UK; ${ }^{e}$ Clinica Universidad de Navarra, CIBERES, Madrid, Spain; ${ }^{f}$ Center for Applied Medical Research (CIMA), University of Navarra and CIBERONC and IdisNa, Pamplona, Spain; ${ }^{\mathrm{g} H}$ Hospital Doce de Octubre and CIBERONC, Madrid, Spain; ${ }^{\mathrm{h}}$ National Cancer Institute of Milan, Milan, Italy; 'University of Clermont-Auvergne, Clermont-Auvergne, France; ${ }^{j}$ ABRCAdaBRA, Milan, Italy; ${ }^{k}$ Centre Leon Bérard and Université Claude Bernard Lyon 1, Lyon, France; 'Institut Bergonié Cancer Center, Bordeaux, France; mUniversità Federico II di

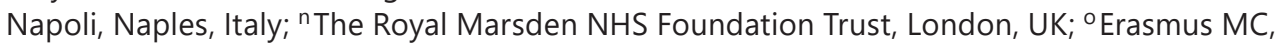
Rotterdam, The Netherlands; ${ }^{\text {P}}$ Aberdeen University, Aberdeen, UK; ${ }^{\mathrm{q}} \mathrm{F}$. Hoffmann-La Roche

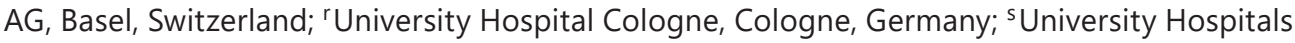
KU Leuven, Leuven, Belgium; ${ }^{t}$ Skane University Hospital, Malmö, Sweden; uUniversity La Sapienza, Rome, Italy; ${ }^{\vee}$ Grosshadern University Hospital, Ludwig-Maximilians University,

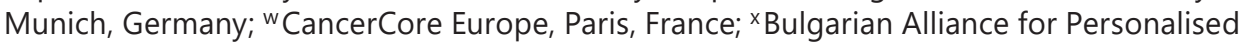
Medicine, Sofia, Bulgaria; yNovartis UK LTD, London, UK; ${ }^{z}$ Istituto dei Tumori Giovanni Paolo II, Bari, Italy; ${ }^{A}$ The European Institute for Innovation through Health Data (i HD), Gent, Belgium; ${ }^{B}$ European Federation of Clinical Chemistry and Laboratory Diagnostics, Milan, Italy; ${ }^{C}$ Eli Lilly and Company, Madrid, Spain; ${ }^{D}$ Digestive Cancers Europe, Brussels, Belgium; EUniversity of Nantes, Nantes, France; ${ }^{F}$ Spanish National Cancer Research Centre (CNIO) and CIBERONC, Madrid, Spain; ${ }^{G}$ EuroScan International Network, Cologne, Germany; ${ }^{\mathrm{H}} \mathrm{BIOEF}$, Basque Foundation for Health Innovation and Research, Barakaldo, Spain; 'Myriad Genetics, Zurich, Switzerland; 'Europa Uomo, Antwerp, Belgium; ' ${ }^{\prime}$ University College Dublin, Dublin, Ireland; LIndependent Patient Advocate, London, UK; ${ }^{M}$ European Institute of Oncology (IEO), Milan, Italy; ${ }^{N}$ Coalition of Healthcare Association, Zagreb, Croatia; ${ }^{\circ}$ Antwerp University

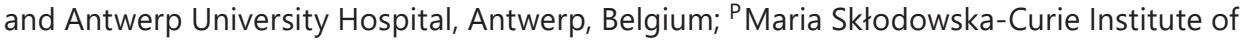
Oncology, Warsaw, Poland; QAstraZeneca, Cambridge, UK
} 


\section{What Is It about?}

Increased molecular understanding is transforming the prospects for healthcare, and molecular diagnostics are opening the pathway to a shift in healthcare strategy. Molecular testing has informed some major preventative public health strategies - such as the significant reduction in cancer mortality. Early diagnosis and personalised healthcare will have a central part to play, particularly in the cancer arena. The paper is the result of extensive engagement with experts and decision makers to develop the framework, and consequently represents a wide consensus of views on how healthcare systems should respond from push and pull factors at local, national and cross-border and EU level.

\section{Keywords}

Biomarkers · Cancer plan · Companion diagnostics · Comprehensive genomic profiling ·

Diagnostic tests · Million European Genome · Molecular diagnostics · Personalised

healthcare $\cdot$ Public health $\cdot$ Reimbursement challenges

\section{Abstract}

Rapid and continuing advances in biomarker testing are not being matched by take-up in health systems, and this is hampering both patient care and innovation. It also risks costing health systems the opportunity to make their services more efficient and, over time, more economical. This paper sets out the potential of biomarker testing, the unfolding precision and range of possible diagnosis and prediction, and the many obstacles to adoption. It offers case studies of biomarker testing in breast, ovarian, prostate, lung, thyroid and colon cancers, and derives specific lessons as to the potential and actual use of each of them. It also draws lessons about how to improve access and alignment, and to remedy the data deficiencies that impede development. And it suggests solutions to outstanding issues notably including funding and the tangled web of obtaining reimbursement or equivalent coverage that Europe's fragmented health system implies. It urges a European evolution towards an initial minimum testing scenario, which would guarantee universal access to a suite of biomarker tests for the currently most common conditions, and, further into the future, to an optimum testing scenario in which a much wider range of biomarker tests would be introduced and become part of a more sophisticated health system articulated around personalised medicine. For exploiting genomics to the full, it argues the need for a new policy framework for Europe. Biomarker testing is not an issue that can be treated in isolation, since the purpose of testing is to improve health. Its use is therefore always closely linked to specific health challenges and needs to be viewed in the broader policy context in the $\mathrm{EU}$ and more widely. The paper is the result of extensive engagement with experts and decision makers to develop the framework, and consequently represents a wide consensus of views on how healthcare systems should respond from push and pull factors at local, national and cross-border and EU level. It contains strong views and clear recommendations springing from the convictions of patients, clinicians, academics, medicines authorities, HTA bodies, payers, the diagnostic, pharmaceutical and ICT industries, and national policy makers. 
Table 1. Snapshot

Some of the current barriers are technical, some are financial, and some are policy-related. This paper sets out what the immediate opportunities are, where the main obstacles are, how they might be overcome, and who stands to benefit - even from the wider provision of only a minimum service. And it looks beyond the immediate horizons, to how optimal use of biomarker technologies could revolutionise European healthcare.

(A summary of short-term Europe-wide solutions appears at the end of Conclusion)

\section{Overview}

Europe is missing out on major opportunities. The rapid growth in the availability of techniques - known as biomarker testing - has made it possible to improve European citizens' health. Their widespread adoption would support continued improvements in outcomes for diseases such as cancer. And the prospects are even more attractive if the development and deployment of more sophisticated biomarkers is encouraged.

But these opportunities are not being grasped. For the sake of patients, and for healthcare systems, it is time to do so (Table 1 ).

The paper identifies important actors needed to secure these benefits. Its perspectives reflect the views of patient advocacy groups (PAGs) and patient representatives as well as the medical and scientific community.

The discussion took place under the umbrella of the European Alliance of Personalised Medicine (EAPM). The mix of EAPM members provides extensive scientific, clinical, caring and training expertise in personalised medicine and diagnostics, across patient groups, academia, health professionals and industry. The discussion reflects this diverse membership and takes account of the new prominence that the use of testing has acquired in providing crucial information in the context of the COVID-19 pandemic. This attention demonstrates the importance of comprehensive, resilient testing services. But the significance of testing is not confined to any specific disease. While all stakeholders are working tirelessly to bring about its implementation as part of an EU-wide health strategy, it is clear that gaps-and-needs are still plentiful, and the challenge is, firstly, to identify them and, secondly, to fill the gaps and meet those needs. Therefore, we need to be able to identify existing barriers in countries across Europe. This is, of course, easier said than done. As stated, PM is in many ways a complex approach to healthcare as it requires changes, often at a base level. The provision, understanding and implementation of new forms of information into clinical practice is just one example. In this instance, the complexity lies in characteristics inherent in the additional data that healthcare professionals (HCPs) will need to employ to its full potential to gain more access for their patients to early diagnosis, treatment and better quality of life.

It has implications for many other diseases, particularly in oncology. Integrating biomarkers as an essential component of oncology treatment and drug development has been crucial to the approval, uptake and use of an ever-increasing number of new personalised medicinal therapies by both the European Medicines Agency (EMA) and the US Food and Drug Administration (FDA). And the health of entire populations stands to be improved by wider deployment of advanced biomarkers.

However, the development and use of biomarkers faces many challenges. Testing is a discipline, a technology of its own, and irrespective of the disease category or type of test, its effective use requires the resolution of some fundamental systemic issues. The outcome of this consensus document makes clear that the emphasis in modern healthcare has shifted significantly in recent decades. More and more we are fighting chronic diseases, and there have been major changes in the way care is delivered. Personalised medicine is geared 


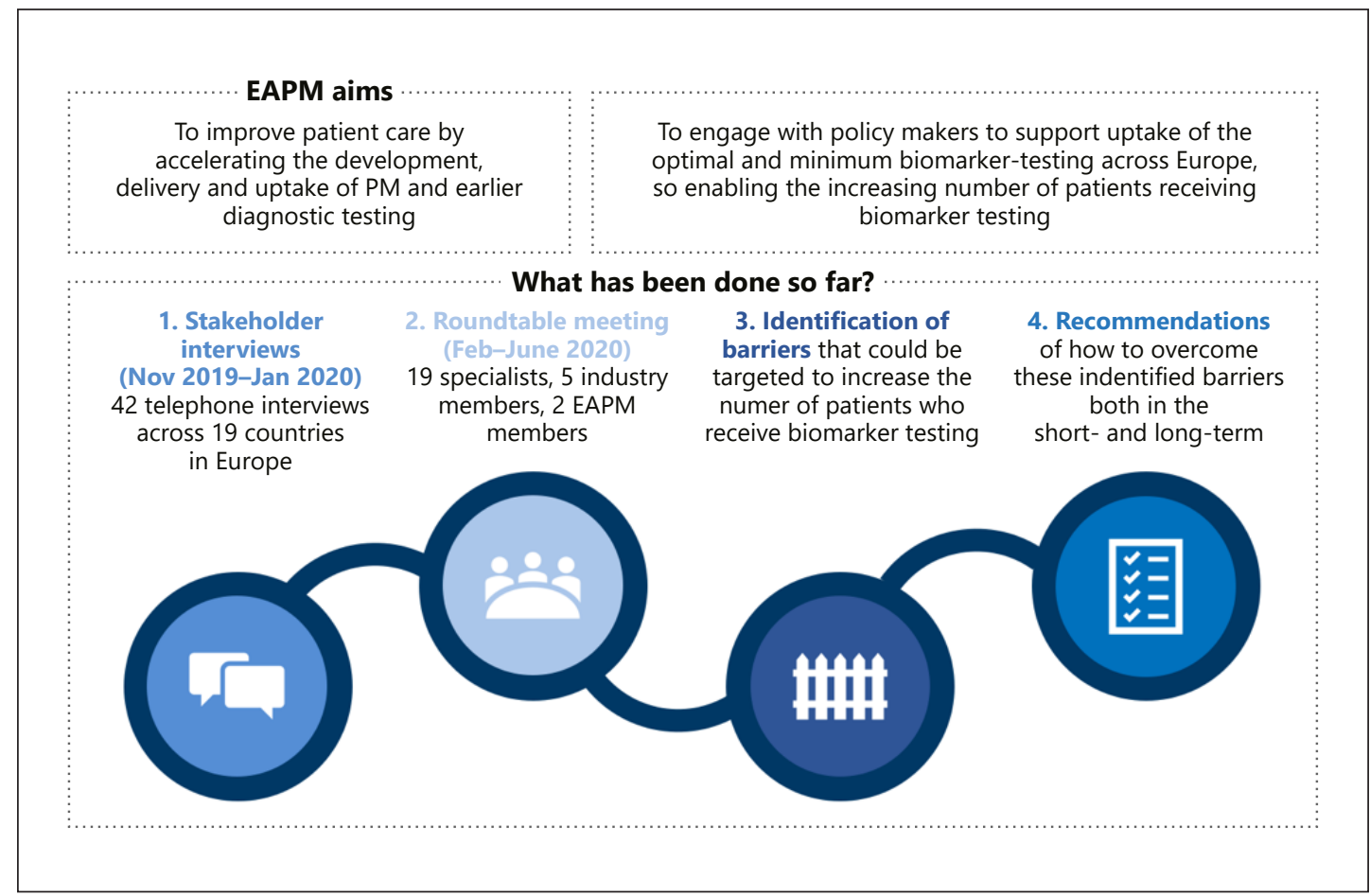

Fig. 1. Evaluating the current biomarker testing landscape could improve Europe-wide access by identifying and targeting barriers.

towards individual patient outcomes and has been defined as a targeted approach to the prevention, diagnosis and treatment of disease based on an individual's specific profile. Biomarker testing and molecular diagnostics are central pillars of this.

From a policy perspective, this means exploring the conditions that are required for biomarkers to play their role now, and to develop their role for the future (Fig. 1).

\section{What Biomarkers Are, and What They Do}

Biomarkers provide information that is of value for patients, HCPs, health systems and society. They constitute a wide range of mechanism and in their purpose, but they all aim to improve and protect health. At the simplest level, they are indicators of health status - running a temperature as evidence of a fever - but the most sophisticated are based on analysis of blood, bodily fluids or tissue, through techniques such as molecular diagnostics including next-generation sequencing (NGS) or artificial intelligence-based measurements. In medicine, biomarkers have multiple functions. A blood or a tissue biopsy, can provide more precise diagnosis to segment the patient's disease into specific group to understand prognosis or predict likely response to a range of treatments, particularly in the complex field of oncology, where biomarkers serve frequently in defining patient populations and with it their likely best treatment. In some cases, biomarkers can provide predictive and prognostic information. They can allow clinicians to foretell the pattern of development in a given individual once a disorder has been diagnosed and can monitor progression. They can also determine an individual's increased likelihood of developing a particular disorder due to an inherited risk or other factors. The process of biomarker development has been driven largely by the urgency 


\section{Biomedicine Hub}

\begin{tabular}{|c|c|}
\hline iomed Hub 2020;5:511209 & \\
\hline DOI: $10.1159 / 000511209$ & $\begin{array}{l}\text { (c) } 2020 \text { The Author(s). Published by S. Karger AG, Basel } \\
\text { www.karger.com/bmh }\end{array}$ \\
\hline
\end{tabular}

Horgan et al.: Bringing Greater Accuracy to Europe's Healthcare Systems: The Unexploited Potential of Biomarker Testing in Oncology

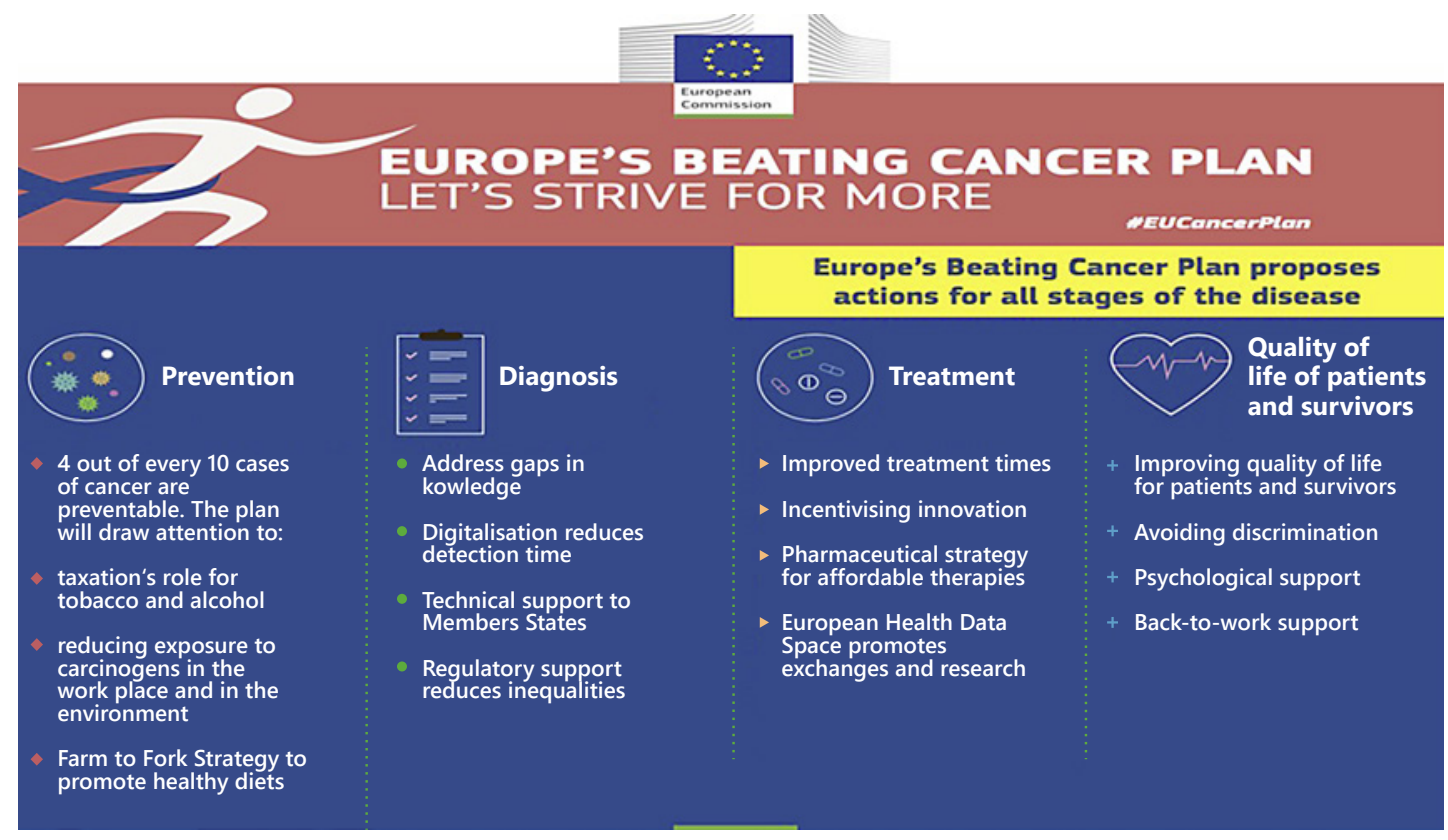

Fig. 2. European Commission's Beating Cancer Plan.

of tackling cancer in all its complexity, not only - if most obviously - because of the huge damage inflicted on patients and their families, but also because of the burden imposed on health systems and more broadly on the economy. The evolution of early screening, diagnosis and treatment is critical to enable better care and outcomes for patients and ultimately less burden on healthcare systems. The Europe Commission's Beating Cancer plan, with its focus on prevention, early diagnosis, treatment and follow-up care, offers a conducive context for directing new attention to biomarker development and use (Fig. 2).

In some instances, biomarkers can be used in large-scale screening for preventive, diagnostic, prognostic or treatment-response predictive purposes to identify people that need special attention. Some have different applications in different contexts - diagnostic in one person and predictive in another, for example, and different approaches can deliver increasingly sensitive results [1].

\section{Types of Biomarkers}

Predisposition Biomarkers

A genetic predisposition (also called genetic susceptibility) is an increased likelihood of developing a health disorder based on the presence of a particular genetic biomarker (e.g., specific mutations in BRCA1 and/or BRCA2 genes indicate that a patient has an increased susceptibility to a group of cancers). Genetic testing for disease risk is often considered when someone has a personal or family history that suggests an inherited risk for a particular health condition, and when the results provide information that can help guide a person's future medical care. It is generally used in asymptomatic subjects. Some other non-hereditary biomarkers are being developed to identify asymptomatic individuals with higher risk of developing a specific type of cancer. Not all predisposition biomarkers are genetic susceptibility biomarkers: chronic inflammatory conditions and their related biomarkers may help to identify individual at higher risk of cancer.

\section{Karger'}


Horgan et al.: Bringing Greater Accuracy to Europe's Healthcare Systems:

The Unexploited Potential of Biomarker Testing in Oncology

Diagnostic Biomarkers

Diagnostic biomarkers are used to confirm that a (usually symptomatic) patient has a particular health disorder. For example, the presence of mutations in the CFTR gene indicates that a newborn has cystic fibrosis. A pathogen-specific test (e.g., HIV) might be used to indicate the presence of an infectious disease. Diagnostic biomarkers may facilitate earlier detection of a disorder than can be achieved by physical examination of a patient. A sub-category of diagnostic markers is screening markers, which differ in that they are typically used in broad asymptomatic populations (e.g., prostate cancer-specific antigen [PSA] for prostate cancer screening, FOBT for colon cancer). CEA is used in colorectal cancer, and AFP and hCG in testicular germ cell tumours. Another sub-category is monitoring biomarkers used to track residual disease (e.g., circular tumour DNA identified in or circular cell free DNA (ccfDNA) or BCR-ABL testing for leukaemia). Diagnostic biomarkers include prognostic (A) and predictive (B) biomarkers:

(A) Prognostic Biomarkers. A prognostic biomarker helps indicate how a disease may develop in an individual when a disorder is already diagnosed. The determination of the presence, absence or altered levels of a prognostic marker can be useful for the selection of patients for treatment but does not directly predict the response to a treatment. Leading examples include Oncotype and Endopredict for early stage breast cancer.

(B) Predictive Biomarkers. A predictive biomarker helps determine which patients are most likely to benefit from a specific treatment option (e.g., Her2 testing in breast cancer for predicting response to drugs such as Herceptin, EGFR mutation or AKL or ROS1 or RET for lung cancer or PDL1 testing in various malignancies or B-raf testing for melanoma). Predictive diagnostics can provide information about how well a treatment is likely to work in a particular patient or about the likelihood of that treatment causing an unwanted side effect. Key subsets of predictive biomarkers are companion diagnostics which have received regulatory clearance for use with a particular drug.

Frequently biomarkers overlap in their clinical use, i.e. predisposition markers may equally be used as predictive markers (i.e., BRCA mutations in ovarian and breast cancer) and predictive markers may also be prognostic (i.e., EGFR mutations in lung cancer).

One overarching goal is to bring greater precision to medicine, which allows greater individualisation of care, and has wide beneficial impacts on population health and on the sustainability of health systems. Subdividing a disease into more specific subgroups can allow more refined and personalised treatment. This improves patient outcomes and drives efficiency in healthcare, reducing reliance on trial-and-error methods and one-size-fits-all approaches, and minimises debilitating toxicities from inappropriate treatment [2].

Personalised medicine and targeted biomarkers have the potential to revolutionise not only the treatment but also the prevention of malignancy, and advances in targeted therapies have changed clinical practice for some diseases. For HCPs, biomarker testing provides a deeper analysis of the disease to differentiate the diagnosis and provide direction on the application of specific treatments. For patients, it expands the chances of successful treatment, provides knowledge about their condition, and widens and allows for stratification of the choices after diagnosis. And for policy makers, it can identify and reduce disease risk, through prevention, early detection and treatment identification, as well as monitoring the effectiveness and choice of treatments. For the health-care system as a whole, biomarkers could make it possible to avoid using new and often expensive drugs in patients that are not going to benefit from them, thus contributing to health system sustainability [3]. It can also contribute to reduce waste, e.g. via avoidance of unnecessary administration of antibiotics. The effective use of biomarkers can contribute to a two-fold objective: maintaining health system sustainability, while providing high value care. For some diseases, biomarkers serving as molecular targets for Precision Medicine have totally 
Horgan et al.: Bringing Greater Accuracy to Europe's Healthcare Systems:

The Unexploited Potential of Biomarker Testing in Oncology

Fig. 3. Main areas of barriers.

\begin{tabular}{|l|l|}
\hline Section & Subsection \\
\hline \multirow{5}{*}{ Science } & System biology and data \\
\hline \multirow{2}{*}{ Operational } & Research \\
\hline & Clinical study design \\
\hline & Information delivery \\
\hline \multirow{5}{*}{ Economics } & Education and training \\
\hline Inform, educate, empower patients \\
\hline Regulations \\
\hline Reimbursement \\
\hline Single technology evaluation \\
\hline Costs of testing \\
\hline Bio-banks \\
\hline Translation \\
\hline Reimbursement \\
\hline Legal and ethical \\
\hline
\end{tabular}

changed overall survival and outcome in formerly devastating cancer diseases, i.e. brc/abl in chromic myeloid leukaemia, c-kit mutations in gastrointestinal stromal tumours or EGFR mutations in lung cancer.

\section{Hurdles to Exploitation}

The actual and potential benefits are obvious. But there are hurdles to overcome both to maximise the use of the current armoury of biomarkers, and to open the way forward to exploiting the huge new opportunities as science and technology open new opportunities for biomarker testing. The figures below provide an overview of the main barriers in terms of science, operations, economics, and the EU-level infrastructure, and of the complex interface of factors in establishing personalised care in the European context.

\section{Main Areas of Barriers (Fig. 3)}

Immediate issues demanding attention range from the quality of testing - which depends also on the quality of the samples being tested (which in turn largely depends on the standardisation of the pre-analytical conditions for sample collection, transport, stabilisation and storage), to the availability of infrastructures for conducting testing, and to skills of responsible professionals in this rapidly advancing field. There are serious challenges to be overcome in the capacity and availability of pathologists and other necessary workforce components in some European countries.

Tests must be analytically and clinically validated to be of any use - a process that is dependent on adequate data, and on the development of more fully agreed and standardised criteria and procedures across Europe and internationally. And many biomarkers need to be re-evaluated over the course of the disease, especially in evolving diseases such as cancer. A framework for cancer management in healthcare systems is a key issue here.

There are challenges too in economic terms - both in agreeing the commissioning or reimbursement of individual tests by the diverse responsible authorities from country to country, and in the overall funding provision for biomarkers in health budgets. The underlying challenge that IVDs face today is that the intrinsic value they generate in terms of infor-

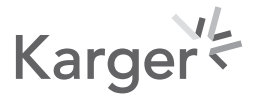




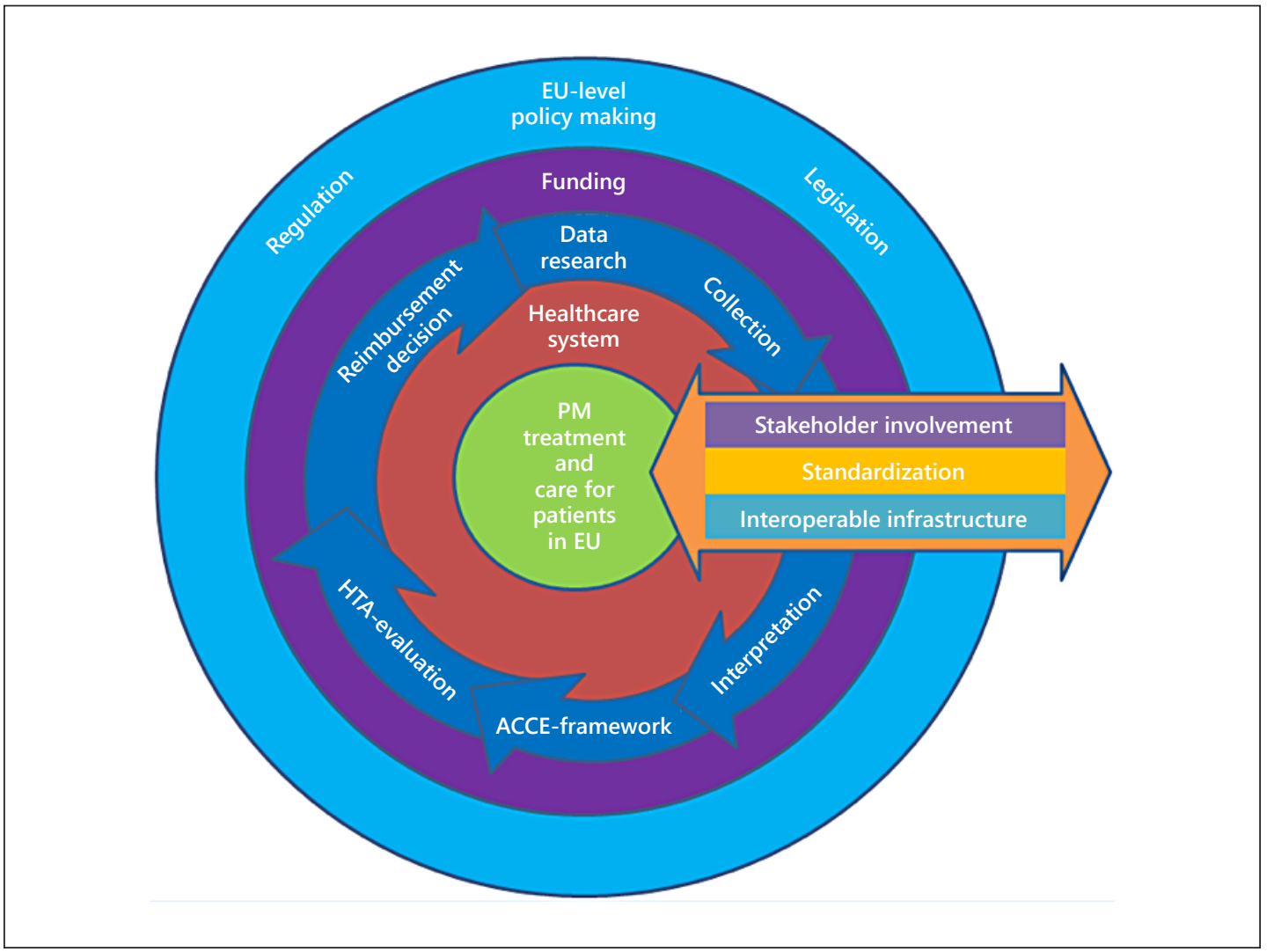

Fig. 4. Index of barriers to PM in Europe [99].

mation is not recognised, nor are they appropriately rewarded on the basis of this value, which has a significant impact on investment in R\&D for innovative IVDs.

Adoption of new tests often requires knowledge and equipment not widely available and depends on the availability of appropriate guidelines from health and medical authorities. The frequent consequence is difficulties of access, compounded by limited awareness among clinicians and patients.

All of these factors in turn play into delays, disincentives and discouragement for taking advantage of the opportunities that biomarkers represent for Europeans' health (Fig. 4).

This paper is designed to offer a review of the opportunities of biomarkers, both immediately and over the mid-term, and to explore the challenges to seizing those opportunities. It aims to present an outline of the questions to be answered, and an outline of some possible solutions.

\section{The Context}

Biomarker testing is not an issue that can be treated in isolation. The purpose of testing is to improve health, so its use is always closely linked to specific health challenges, and needs to be viewed in the broader policy context in the EU and more widely. In the EU, relevant developments include the parallel initiatives of its "Beating Cancer Plan" and its "Cancer Mission" (since it is in the field of oncology that testing is playing an increasing role), the project to create a "European health data space," and the "Pharmaceutical Strategy" that the 


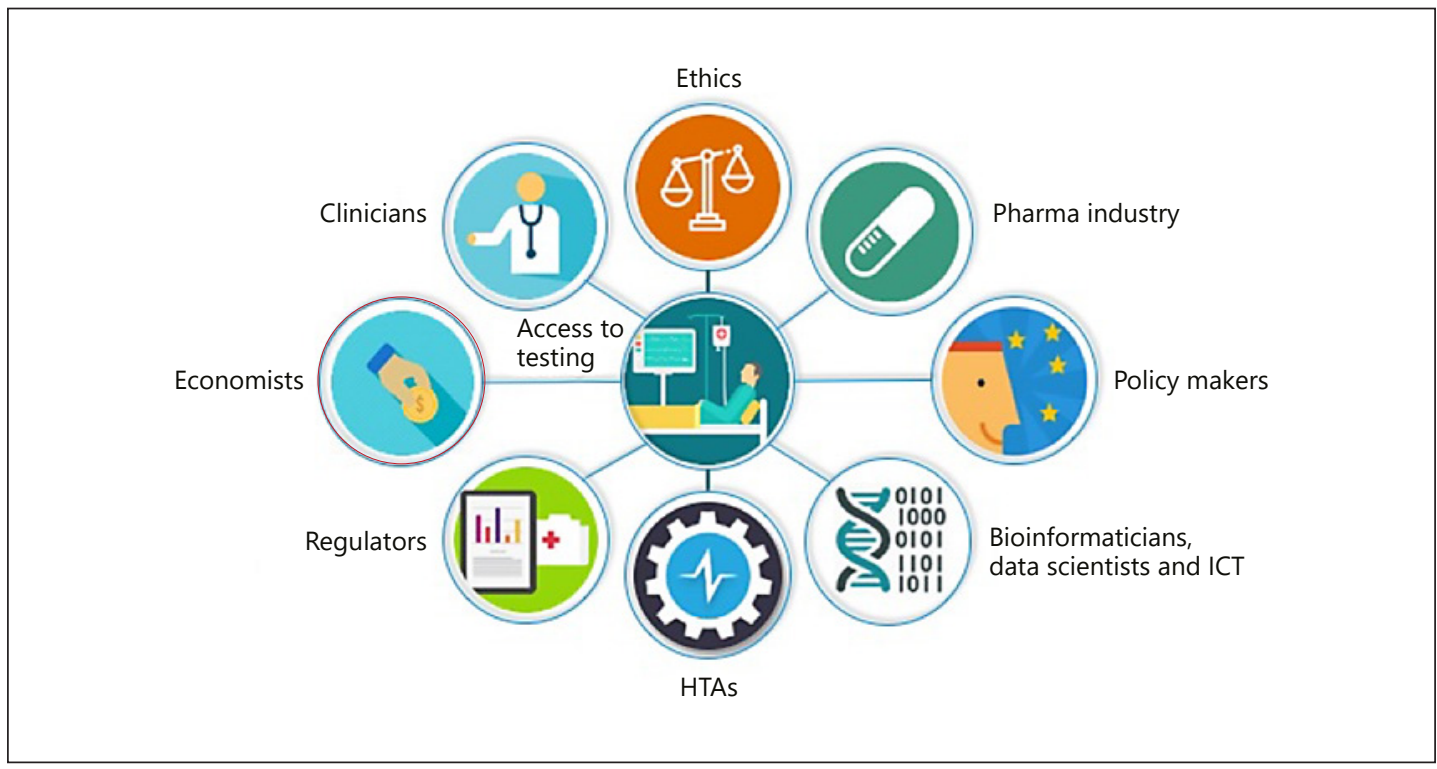

Fig. 5. Involving every stakeholder group is necessary to improve access to testing in the EU.

European Commission is scheduled to deliver at the end of 2020. The evolution of these EU initiatives depends, as always in the EU, on a complex array of interlocking influences and stakeholders: decisions are not made by decree, but are the consequence of extensive and often prolonged debate among advocates of disparate viewpoints (Fig. 5).

The Cancer Plan, now in development, is intended to update the EU's approach to cancer to reflect new knowledge over the last two decades, and the Cancer Mission is designed to maximise the capacities of Europe's research community to push real breakthroughs in cancer care. The potential of advanced biomarker testing has an obvious place in these discussions: molecular diagnostic testing is embedded in cancer treatment pathways - from reliable guidance on optimal treatment for the individual patient, to precise monitoring of its effectiveness [4]. The planned European Health Data Space will provide a framework for the collection and analysis of the data that is a condition for the essential task of measuring the impact of the use of a given biomarker [5]. At the same time, the Pharmaceutical Strategy, the first such exercise for more than a decade, will attempt to forge greater EU level coordination and collaboration in facing the key challenges that now face EU pharmaceutical policy [6]. The outcome of all these reflections should give due recognition to the role of biomarkers, including by providing a policy framework in which member states would find it easier to reach consistent decisions and to provide clearer funding arrangements, thus boosting access and continued development (Fig. 6).

In such a rapidly evolving technology, the international dimension must also be taken into account - and particularly the US context, where much biomarker innovation is taking place. By comparison with the EU, where the implementation of new (but already belated) regulations on in vitro diagnostics and medical device regulation (IVDR and MDR) has been partly delayed, some countries provide regulations that guarantee a more rapid deployment of new techniques. [7] With respect to CDx, the FDA has developed processes for the qualification of biomarkers, and diagnostic tests that measure a biomarker may follow the established regulatory decision processes for evaluating companion diagnostics, or even be dispensed from that obligation in the case of a biomarker for a single drug development programme. In vitro diagnostics are regulated by the FDA and Japan's Pharmaceuticals and 


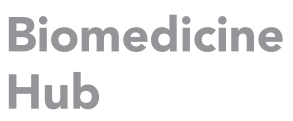

\begin{tabular}{|c|c|}
\hline \multicolumn{2}{|l|}{ Biomed Hub 2020;5:511209 } \\
\hline DOI: $10.1159 / 000511209$ & $\begin{array}{l}\text { (c) } 2020 \text { The Author(s). Published by S. Karger AG, Basel } \\
\text { www.karger.com/bmh }\end{array}$ \\
\hline
\end{tabular}

Horgan et al.: Bringing Greater Accuracy to Europe's Healthcare Systems:

The Unexploited Potential of Biomarker Testing in Oncology

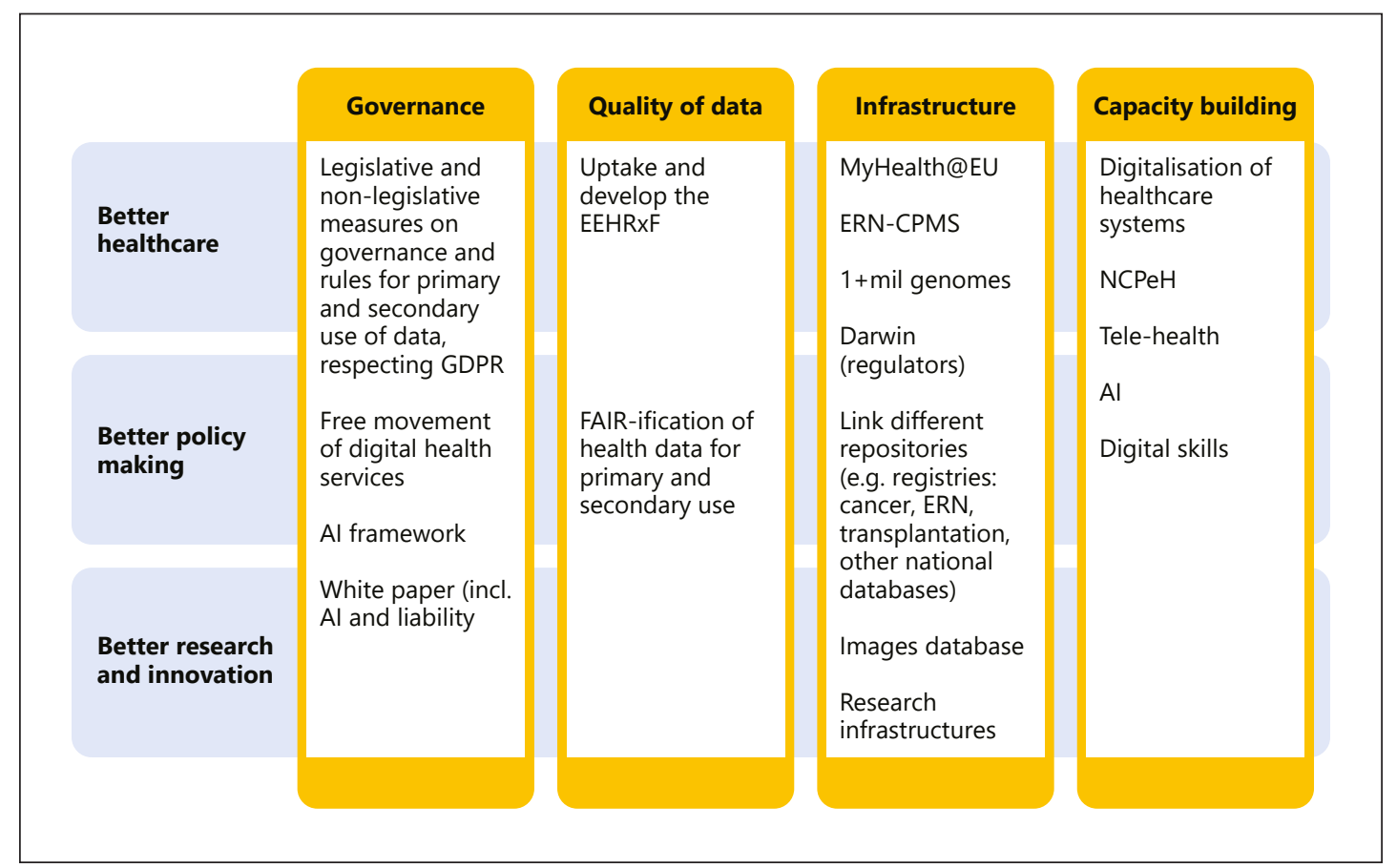

Fig. 6. Matrix of the E-Health Data Solutions.

Medical Devices Agency (PMDA) [8]. When novel targeted therapies are approved by the FDA and the PMDA, there is a close link with the approval of related companion diagnostic tests. This is of course something of a special case. Even if, in due course, the same comes to apply in Europe, it is only one among a range of possibilities. Meanwhile, Europe faces particular difficulties, as outlined below [7]. Regulatory approval is only the first (yet very important) stage in the access pathway for biomarkers. Other hurdles include reimbursement and assessment (effectiveness, added value), especially when it comes to innovative biomarkers. Challenges for equal access to companion diagnostics in Europe relate to the variation of approaches for assessment and reimbursement across and within Member States, the general lack of link between assessments and decision making, and the lack of established clear processes for reimbursement, funding and uptake of diagnostic innovation. Access decisions are often based on different and imprecise processes, procedures and requirements, slowing adoption of companion diagnostic tests and subjecting the tailoring of clinical and economic evidence to uncertainty. And even where the funding mechanisms ensure these tests are reimbursed by insurance companies or healthcare providers immediately, it remains a major struggle to integrate multiple biomarkers into multi-gene panels. The uncertainty on access, funding and uptake of molecular diagnostics, such as companion diagnostics, has a direct negative influence on investment decisions, further hampering progress and the integration of innovation. The long-drawn-out and still non-applied modernisation of relevant EU legislation - with its definition of companion diagnostics and its new challenges in shifting from a positive list approach to a more flexible risk-based approach, raising issues of global alignment, scientific and analytical validity, and clinical performance - has done little so far to ease the challenges (Fig. 7; Tables 2-4).

Against this background, this paper aims to stimulate discussion on how the energy behind ongoing EU policy moves can serve to make the best use of current biomarkers, and to drive forward innovation in the field. This ambition has two aspects: a pragmatic agenda 
Horgan et al.: Bringing Greater Accuracy to Europe's Healthcare Systems: The Unexploited Potential of Biomarker Testing in Oncology

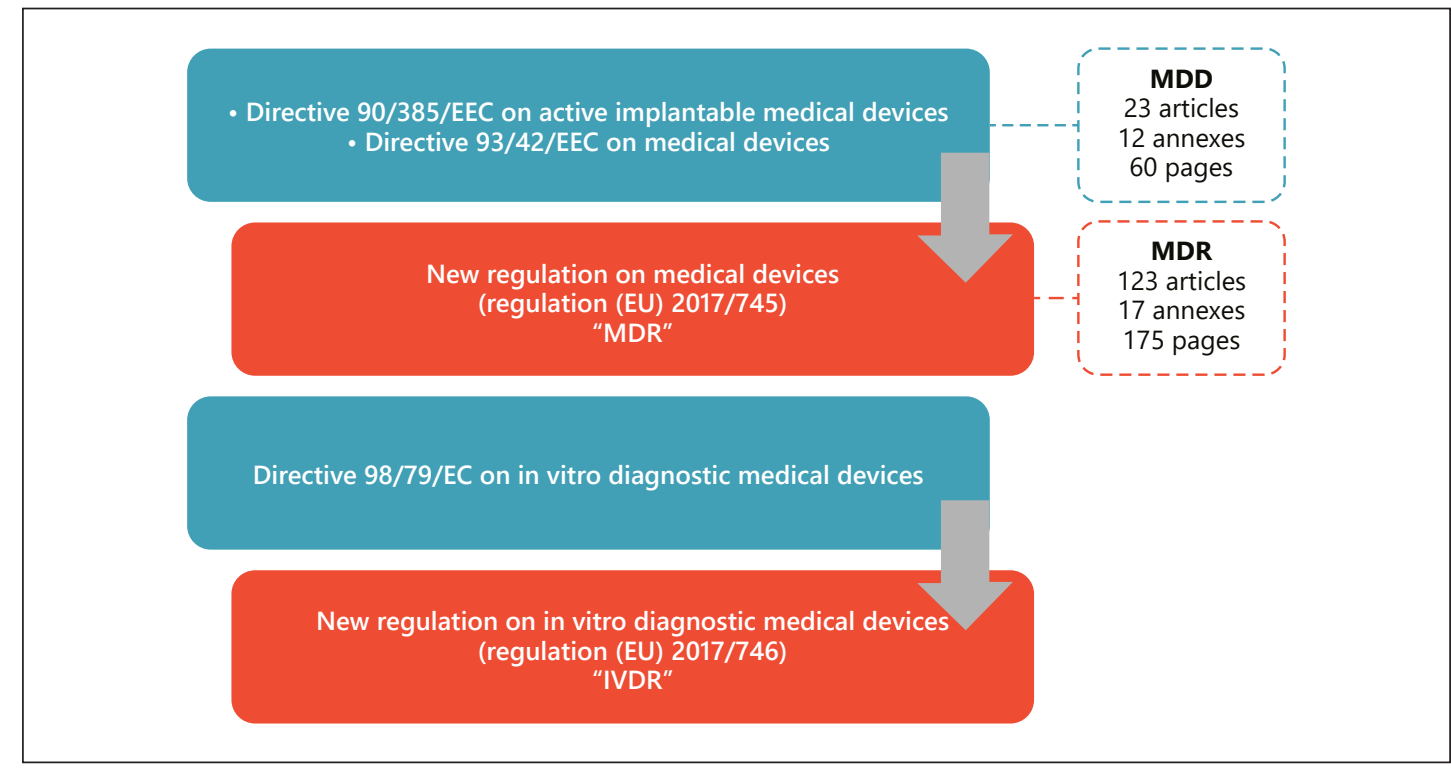

Fig. 7. Revision of the EU medical devices legislation. Adopted on April 5, 2017, publication in OJ-EU, May 5, 2017.

Table 2. Companion diagnostics: new EU definition

Art. 2(7) IVDR

"Companion diagnostic" means a device which is essential for the safe and effective use of corresponding medicinal product to: (a) Identify, before and/or during treatment, patients who are most likely to benefit from the corresponding medicinal product, or (b) identify, before and/or during treatment, patients likely to be at increased risk of serious adverse reactions as a result of treatment with the corresponding medicinal product.

IVDR Article 48(3)

For companion diagnostics the notified body shall consult the concerned competent authority designated in accordance with Directive 2001/83/EC or the European Medicines Agency (EMA), as applicable.

ANNEX IX, Chapter II - 5.2.

The notified body shall, before issuing an EU technical documentation assessment certificate for the companion diagnostic and on the basis of the draft summary of safety and performance [...] consult one of the competent authorities [...] regarding the suitability of the device in relation to the medicinal product concerned.

for immediate improvements, and - more idealistically - developing a vision of what dramatic further contributions biomarker testing could deliver with greater resources and policy commitment.

Already, in 2020, a broad arsenal exists of valuable biomarkers, but earlier policy decisions continue to influence - and in some ways hinder - their deployment even in generating a minimum level of provision, and it is possible to identify many of the changes needed to overcome unwarranted obstacles. At the same time, the current armoury is also an eloquent herald of what could be achieved by the deployment over the medium term of the more advanced biomarkers now emerging or in imminent prospect. The realisation of these possibilities depends largely on policy choices: Europe can install wide access to high-quality testing with a minimum level of service. It can also choose to begin a move beyond a merely

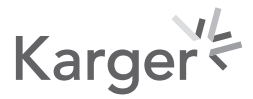


Table 3. The EU's new In Vitro Diagnostic Regulation

- The EU's new In Vitro Diagnostic (IVD) Regulation (IVDR) applies on the 26th May 2022 and replaces decades old legislation. This new regulation will require commercial IVD manufacturers, who wish to market their IVDs across the EU, to comply with stringent requirements relating to product development, performance evaluation, data reporting and quality assurance. In addition, the EU IVDR will impact public and private laboratories, who produce lab-developed tests (LDTs) or "in house production" tests, as there will be a requirement to justify their development and use where commercially available tests exist. Where a single healthcare institution proceeds with offering an LDT, they will be required to meet all the relevant requirements for safety and performance, providing a justification for not using a commercially available CE-marked IVD, carrying out the manufacture under an appropriate quality management system and making publicly available certain details of the LDT.

- As a result, IVD manufacturers producing commercially available CE-marked IVDs can expect higher costs and longer timelines for developing new products, as well as costly new clinical monitoring and evidence generation to recertify many existing products. Furthermore, in many cases, public labs, relying upon the development and use of LDTs across their health organisation, may be required under the IVDR to utilise the commercially available CE-marked IVD.

- The regulation will take effect across all member EU states on 26 May 2022, profoundly affecting business and operating models of all IVD companies and public labs.

Table 4. Recommendations

- The European Commission should consider a 12-month postponement of the date of application of the IVDR to mitigate capacity challenges within the notified bodies and the effect of COVID-19 on European stakeholder's preparations for this new regulation. This would be in line with a recent decision for a 12-month postponement of the MDR.

- The European Commission's Medical Devices Coordination Group and the member states' National Competent Authorities for IVDs should develop clear guidance for public and private laboratories on how they should move forward with the development of lab-developed tests under the new regulation.

Table 5. Recommendations to the EU

- The European Commission to update by 2021 its recommendation on early detection strategies allowing for risk stratification through molecular diagnostics/biomarker testing.

- European health authorities to put in place a policy framework to support diagnostics in the EU by 2022, with a ring-fenced budget allowance for biomarker testing development (clinical validation). Research on biomarkers discovery and early testing should also be a matter of concern as well as adequate funding.

- The EU to agree by 2023 a business model for public-private cooperation for optimal biomarker testing available across the EU.

- $\quad$ The EU to provide guidance on minimal testing standards and resource allocation by 2025.

- The EU to establish a framework for quality of testing and value of diagnostics information, with a fast track approval mechanism for biomarker validation.

- The EU to facilitate centralised and standardised registries of diagnosis including sequence and biomarker data, pre-analytical sample metadata, treatment and outcome.

pragmatic approach, and aim for an ideal level of provision in an optimum setting - embracing the accelerating pace of science and technology, so as to provide increasingly sensitive results and still greater healthcare improvements. There are, in effect, two questions: what policy framework could ensure a high-quality minimum biomarker testing context across the EU in 


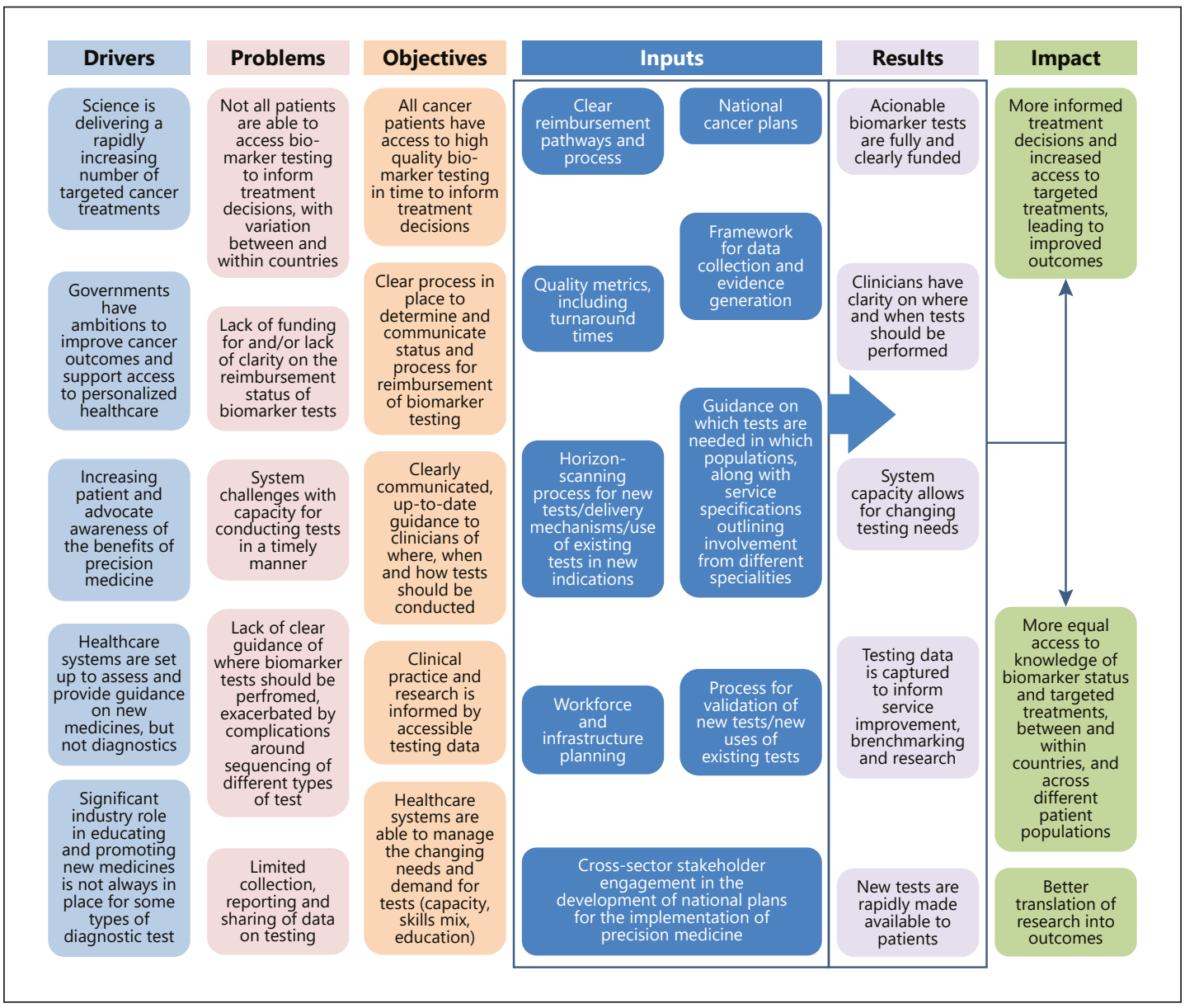

Fig. 8. Minimum testing: drivers, problems, objectives, inputs, results and impact.

the short term, and the longer-range question of how health might be improved in a scenario of optimum biomarker testing availability [9].

The minimum and optimum: in both paradigms, the aim is to secure the best standard of care for patients, but within the objective limitations of resources, capital (human, financial, infrastructure, etc.) from country to country, region to region, even hospital to hospital. The perfect scenario where resources were not an issue would be the optimum. The minimum would be the basic testing on actionable biomarkers that should be made available across the EU in the immediate future, accompanied by efforts to scale up towards the optimum as quickly as circumstances permit. This paper does not seek to identify specific tests that should be provided in each scenario, but to set out the policy and healthcare infrastructural requirements that would be needed to support these two paradigms (Table 5).

\section{Minimum Testing}

The initial and immediate need is for a framework that provides a minimum level of biomarker provision across Europe - an "essential package" on the testing that needs to be offered to all EU patients for diagnosis and prevention over the next 5 years, detailing the type, the technique and the methods of implementation, and ensuring access to high-quality 


\section{Biomedicine Hub}

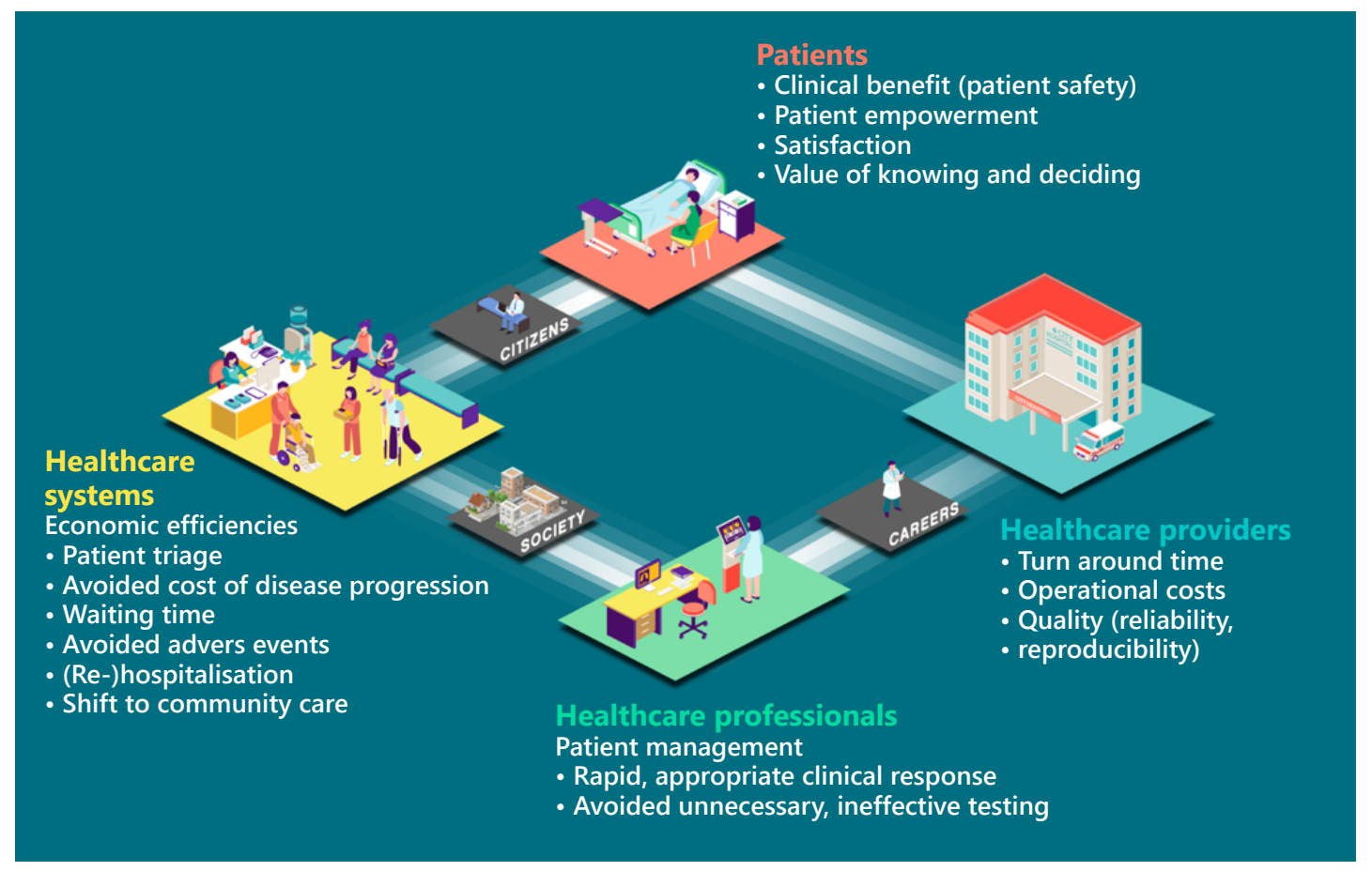

Fig. 9. VODI - value of diagnostic information.

biomarker testing in time to inform treatment decisions, backed by clarity over reimbursement, and up-to-date testing guidance to clinicians. This would be a definition of minimum testing standards and provision, so that currently available technology and knowledge is used to deliver health gains to patients and families across Europe. This would lead to actionable biomarker tests that are fully and clearly funded, to clarity for clinicians on where and when tests should be performed within a system capable of allowing for changing testing needs. Testing data would be captured to inform service improvement, benchmarking and research. And new tests would be rapidly made available to patients $[3,10]$.

Figure 8 offers a schematic summary of what a minimum service in biomarker testing might bring - and of the steps that would be necessary to achieve it.

The impact: more informed treatment decisions and increased access to targeted treatments, with improved outcomes. And in parallel to issues of treatment, biomarker testing has value along the whole pathway, prevention to monitoring. There would also be more equal access to knowledge of biomarker status and targeted treatments, between and within countries, and across different patient populations - with clearer understanding of value of diagnostic information (VODI) (Fig. 9).

Even a cursory glance suggests that this minimal objective is still far from being achieved. In cancer, which is the disease area best served at present by genomic/molecular biomarkers, a minimum testing scenario should guarantee access for all patients to high-quality testing for actionable biomarkers in time to inform treatment decisions. But there are wide variations in provision across technologies, disease areas, patient populations, and across Europe, despite the targeted cancer treatments that science is delivering [11], and most importantly early detection and even prevention. Europe's apparatus for product approval of biomarkers is under-developed, lacking the clearer procedures - and related support - for obtaining drug approvals. Uneven availability of biomarker testing from country to country - or hospital to hospital - inhibits treatment decisions and is compounded by the uncertainties over reimbursement status for biomarker 
Table 6. Short-term solutions for Member States

- In the short term, Europe should provide widespread, consistent access for cancer patients to clinically meaningful testing results based on long-term validated biomarkers (e.g., EGFR, HER2, ALK, etc.).

- It should offer "essential packages" on the testing (type, technique and methods of implementation) around diagnosis and prevention at an EU level.

- It should recognise the business and value case to define infrastructure to deliver testing requirements.

- It should require validated testing to be provided only by labs that are ISO15189-accredited (or equivalent) and that uphold standards throughout Europe.

- It should set up procedures to capture and share efficiency and outcome data from testing.

- It should build the evidence base on biomarker testing, and define how this is accessed, such as on a pan-cancer registry across the EU.

- It should establish a multi-disciplinary steering group (including patients, evaluators and industry partners) to determine what information needs to be provided to stakeholders (including lab owners) and when, with particular emphasis on what information to give to patients, when and by whom.

- $\quad$ Provide guidelines on how clinically relevant biomarkers should be measured and reported.

tests. Awareness of, expertise in, the level of standardisation, the quality of the sample and capacity for conducting tests are not uniform, and the collection, reporting and sharing of data on testing and time to diagnosis are widely inadequate and heterogeneous across Europe [12].

Policymakers, practitioners and product developers face a barrage of challenges. There is incomplete or deficient knowledge of the potential and the value of biomarkers - and of the implications of their more widespread adoption, both in terms of opportunities for better care, and in terms of the associated health system requirements for their effective deployment, including resources and capacity for timely patient access. While the number and complexity of available tests continues to increase and will continue at even faster pace in the near future, inconsistency persists among evidence frameworks for diagnostics, and among standards for demonstrating clinical utility, which has the effect of perpetuating inconsistency and uncertainty on reimbursement arrangements [13].

In a minimum testing scenario, the objectives should include ensuring that all cancer patients have access to standardised, high-quality clinically validated biomarker testing in time to inform treatment decisions, backed by clarity over reimbursement; and clinicians would be provided with up-to-date testing guidance. As a minimum, healthcare systems across Europe should be able to manage changing needs and demand for tests, and operate horizon-scanning processes for valuable innovations in hardware of tests, software of mechanisms and new definition of patients populations that may benefit from target treatments. Then, equipped with quality metrics, authorities could plan workforce and infrastructure modifications, and provide more refined guidance on testing.

If those inputs were in place, it would lead to actionable biomarker tests that are fully and clearly funded, and to clarity for clinicians on where and when tests should be performed within a system capable of allowing for changing needs. Testing data would be captured to inform service improvement, benchmarking and research. And new tests would be rapidly made available to patients [14].

The impact would be to provide more informed treatment decisions and increased access to targeted treatments, with improved outcomes. There would also be more equal access to knowledge of biomarker status and targeted treatments, between and within countries, and across different patient populations. Better and quicker translation of research into outcomes would also result (Table 6). 


\section{Practical Cases}

Some concrete examples of what can - and could - be done in specific diseases offer illustrations of the merits - and the current limitations - of biomarker testing, in identifying predisposition to disease, in assisting diagnosis and monitoring of disease, in indicating how a disease may develop, and in predicting the likelihood of a patient benefiting from a particular treatment option.

\section{Breast Cancer}

In breast cancer, testing of tumour biopsy now makes it possible to identify certain mutations that drive treatment resistance (notably PIK3CA - which, additionally, is often missed in current routine testing). This already permits better risk assessment of patients, and also assists the search for therapies that specifically target these mutations, so that testing can then help guide treatment decisions [15].

Prognostic testing for early stage breast cancer has been available in the US since 2004, but EU adoption was much delayed. While now partially available in 12 EU countries, such tests are still limited. Multi-gene expression profiling can prognose outcomes in early stage breast cancer, informing decisions over hormonal treatment, chemotherapy, radiotherapy or surgery. This can support clinical decision making in patients with early stage breast cancer by identifying those who may require adjuvant chemotherapy, and those for whom hormonal treatment is adequate [16].

In a minimum scenario, validated tests should be available for patients at the right time for, for instance, PIK3CA-mutated, advanced or metastatic breast cancer. Wider use of NGS should reveal more than the current single gene testing, liquid biopsy could replace the need for tumour tissue testing, allowing identification of variants quickly and with increasing affordability, and testing protocols should be developed to support fully informed treatment decisions for the greatest number of patients [17].

Breast cancer has led the way with prognostic and predictive biomarkers. Long- established predictive biomarkers include oestrogen receptors (ER)-progesterone receptors (PR) for predicting response to endocrine therapy and HER2 for predicting response to anti-HER2 therapy. These predictive biomarkers should be used in the neoadjuvant, adjuvant and advanced disease settings [18]. Newer predictive include BRCA1/2 mutation for predicting response to PARP inhibitors and PIK3CA mutations for predicting response to alpelisib (in combination with fulvestrant). Both these newer predictive biomarkers should only be used in patients with advanced breast cancer [19].

Established and recommended prognostic tests in patients with ER-positive, HER2negative, lymph node disease include the multianalyte tests such as UPA-PAI-1, Oncotype DX, MammaPrint, EndoPredict, Breast Cancer Index (BCI) and Prosigna (PAM50). Oncotype DX, MammaPrint, EndoPredict or Prosigna may be similarly used in patients with 1-3 metastatic lymph nodes. The main use of these tests is in identifying newly diagnosed breast cancer patients whose outcome is so good, that they are unlikely to benefit from adjuvant chemotherapy [20].

\section{Ovarian Cancer}

Ovarian cancer - the deadliest gynaecological cancer - still has limited screening options for early stage diagnosis. If germline mutations are identified in genes such as BRCA1 and BRCA2 that increase the risk of ovarian cancer, patients can undergo riskreducing surgery. This may also support identification of potentially at-risk family members through cascade testing, or the offer to germline testing to the direct family members of a positive patient. 
It is recommended and well accepted to test any new high-grade ovarian cancer patient for mutations, particularly BRCA1 and BRCA2. If a BRCA1/2 mutation is found in a patient (which may be results of either an inherited mutation or a spontaneous mutation on the tumour itself), this information can be used to guide therapy. Since 2008, evidence-based guidelines have recommended that all ovarian cancer patients be tested for BRCA1 and BRCA2 mutations, but relatively few have a recorded test. Testing now impacts the type of treatments for which these patients are eligible, and the low level of BRCA testing in this population means that significant opportunities exist to improve cancer prevention and treatment $[21,22]$.

In advanced ovarian cancer, tumour BRCA (tBRCA) testing can detect both germline (inherited) and somatic (spontaneous) mutations, to identify patients for targeted treatment, and germline BRCA (gBRCA) testing can detect inherited mutations to identify patients for targeted treatment, which can significantly reduce the risk of disease progression or death. Testing is also important for cancer prevention for family members of patients with an inherited BRCA mutation, since they have an increased risk of developing some kinds of cancer.

For the future, add-on tests may complement BRCA to better determine eligibility for targeted treatments [23].

\section{Prostate Cancer}

PSA is a protein almost exclusively produced by the being prostate epithelium and in a majority of prostate cancer cells. Although it is a unique biomarker in blood for early detection of prostate cancer, it has been criticised for not being a specific test at moderately elevated levels. Opportunistic screening with a single PSA test has led to the detection and overtreatment of indolent tumours. The introduction of multiplex test (like PHI, 4K score and STHLM3), with different forms of PSA together with age, previous negative biopsies and family history increase the specificity of detecting significant cancers. Although recommended in guidelines, some of these are currently expensive and not commonly available, but the approach should be factored into refining diagnostic and therapeutic strategies [24]. The decisions on whether a patient at risk needs a biopsy (or a further biopsy) or on the right treatment for a diagnosis can also be assisted by multiparameter magnetic resonance imaging which is considered as an imaging biomarker for significant disease.

Multi-gene expression profiling can prognose the aggressiveness of prostate cancer and can support clinical decision making in patients with indeterminate risk based on other testing data. Commercially available tests are frequently used in the Unites States but not in Europe. They may have a role in treatment decisions but only together with available clinical parameters as recently reviewed by a panel expert initiated by the ASCO guidelines group [25]. These gene profiling tests for prostate cancer require further validation, especially in prospective trials before they can be generally recommended for routine clinical use.

The frequency of somatic mutations is low in newly diagnosed prostate cancer; however, in castration-resistant disease, aberrant gene expression is more commonly found related to the androgen receptor, TP53, SPOP, PTEN and other genes. The well-documented germline mutation - BRCA2 - leading to breast and ovarian cancer is also important for the development of prostate cancer, and it has been integrated in some algorithms on early detection (next to African American origin, familial predisposition and high rapidly increasing PSA) [26, 27]. Genetic testing for BRCA2 and other DNA repair genes is now routinely performed to identify responders to the Poly (ADP-ribose) polymerase inhibitors (PARP) inhibitors (olaparib, rucaparib, niraparib, talazoparib) which illustrates precision medicine in advanced prostate cancer.

For the future, decentralised prognostic test kits could enable in-house testing of blood or urine. Similarly to PARP inhibitors, new drugs need to be implemented with the companion diagnostics in precision medicine [28, 29].

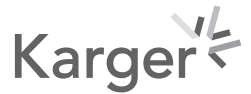




\section{Non-Small Cell Lung Cancer}

In non-small cell lung cancer, where large numbers of actionable genetic abnormalities are susceptible to targeted therapies, the current range of tests for mutations identifies patients that are eligible for targeted therapies. Clinical validation study results have been published for a variety of biomarkers, and make it possible to avoid giving expensive and noneffective drugs to patients that are not likely to respond [30].

Access to targeted therapies for lung cancer depends on accurate identification of patients biomarkers through molecular (mutational) testing, but survey results suggest that many clinicians in several countries are unaware of evidence-based guidelines that support the use of molecular testing. More than 6 of 10 respondents (61\%) report that molecular testing rates are less than $50 \%$ in their country. Nearly 4 in 10 surveyed (39\%) were not satisfied with the conditions of molecular testing in their country, citing concerns with the time it takes to receive results, the reliability of samples and difficulty understanding results. Continuous education around the value of information provided by molecular testing in lung cancer should be intensified at national and international levels to ensure patients receive optimal therapy [31].

A wide range of possibilities are emerging for biomarker testing in defining lung cancer options, ranging from optimised image-based screening, the use of autoantibodies or other circulating biomarkers such as microRNAs, protein panels and other. Sensitivity or specificity of early detection may be improved through panels of cancer antigens measured in serum or in plasma, or even novel NGS of circulating tumour DNA, which could improve diagnostic accuracy [32]. Further ahead, options may include the use of exhaled breath condensate for cancer detection, or enhanced cytology techniques based on sophisticated image analysis algorithms and with artificial intelligence, which could add sputum as an additional useful non-invasive body fluid to identify biomarkers in the context of lung cancer screening [33].

But multiple research challenges face biomarker development and validation in the context of lung cancer screening, where the interaction between genetics and external factors is multidimensional and hard to control. And so many biomarkers are approaching clinical validation that the field is in great need of standardised metrics of clinical utility - as it is across all cancer types [32, 34].

The EU should promote early detection programs (high-risk population screening) of new tumour types, for example lung cancer. Efforts should be placed on an analysis of the potential health benefits of lung cancer screening at the EU high-risk population level. Wellvalidated biomarkers, analysed within the low-dose CT programs, may help to refine cost effectiveness and accuracy of early detection.

The EU and member states should ease regulatory barriers to data transmission and the transport of human samples to permit large international multi-centre clinical validation studies of biomarkers (especially early diagnostic and prognostic biomarkers), which require large and long-lasting cohorts [35].

For the future, the range of testing in the context of drug response-predictive biomarkers should be expanded to cover all actionable mutations. Mutational panels testing should be the preferred choice in the short term as more actionable biomarkers are approved, transitioning to NGS in the long term.

\section{Colorectal Cancer}

Molecular testing for colorectal cancer helps to guide decisions, adapting treatment to targeted therapies to reduce the risk of disease progression or death. Germline testing can also detect inherited mutations to identify patients belonging to families with a high risk of cancer.

For the future, improved clinical, histological, and molecular characterisation of colorectal cancers would allow the development of new targeted therapies, and the use of immuno- 
therapy for microsatellite instability positive colorectal cancer (which is currently available only in therapeutic trials). Circulating tumour DNA identified in ccfDNA analysis could also be used to detect disease progression earlier, leading to different and potentially more effective treatments [36].

\section{Thyroid Cancer}

Several genomic alterations relevant across the different histological types of thyroid cancers are driven by molecular biomarkers, including BRAF, NTRK, mTOR, RAS and RET [37]. Knowing the oncogenic molecular driver helps to determine the aggressiveness of the tumour and/or to identify the most appropriate systemic or targeted therapy. Some molecular markers are indicators for the aggressive behaviour associated with tumour dedifferentiation, including p53, PIK3CA, CTNNB1 and AKT1. Mutation in the telomerase reverse transcriptase (TERT) promoter can be a marker of malignancy as well. BRAF mutation detected through molecular testing may indicate a need to treat the tumour more aggressively. NTRK gene fusion testing can help in guiding treatment of individuals with advanced solid tumours. RET testing for both germline and somatic mutations is helping clinicians to better identify the patients eligible for targeted therapies, and also to prevent the development of thyroid cancer in patients with inherited RET mutation [38].

Despite knowledge of oncogenes (for more than 25 years in respect of RET [39], NTRK [40] and BRAF] [41], biomarker testing is not routinely performed for all of them. This results in part from the focus of guidelines on clinically actionable biomarkers with approved therapies, but also from the gaps in infrastructure and testing methodologies, such as single gene testing or a prioritisation of testing for the most common oncogenes.

Of the various methodologies used to detect genomic alterations, NGS gives the most comprehensive view across a large number of genes, identifying mutations, fusions and other alterations, using a minimal quantity of tissue. However, this is not yet a routine in clinical practice. Very recently, liquid biopsy has presented a new non-invasive source for monitoring cancer genetics in the blood and is taken into consideration in several personalised thyroid cancer diagnosis and prognosis strategies.

- At a minimum in Europe, all metastatic TC patients would be tested at diagnosis for all potential actionable biomarkers (both somatic and germline mutations in the case of medullary thyroid cancer) before treatment decision. Tests would become available and reimbursed on approval of the targeted treatment, with guidelines updated and implemented accordingly.

- In an optimal testing scenario, all thyroid cancer patients would benefit at diagnosis from a routine incorporation of quality comprehensive genomic testing in clinical practice (tissue or liquid samples), the testing results would reach the HCP in time for the treatment decision, and all biomarker tests would be reimbursed. Each thyroid cancer patient should be considered with regard to their genetic background to choose the exact therapeutic component.

Patients with thyroid cancer ideally should discuss the role of genomic-biomarker testing for the management of their cancer with their treating oncologist. The possibility of recognising the genetic signatures associated with a poor prognosis among all the biomarkers identified would represent a valuable diagnostic and prognostic tool to better stratify the risks, prevention and potential use of targeted therapies.

Pan-Cancer

Across the entire range of cancer - "pan-cancer" - there is also rich potential in the integration genomic profiling, matching the right targeted therapy to the detected genetic aberrations, and evaluating treatment outcome. Applications combining testing using NGS with 
Horgan et al.: Bringing Greater Accuracy to Europe's Healthcare Systems:

The Unexploited Potential of Biomarker Testing in Oncology

artificial intelligence and machine learning may ultimately help chart entire clinical pathways $[42,43]$.

This would require a clinical infrastructure that could turn fit-for-purpose real-world data into real-world evidence to help close evidence gaps, overcoming the deficiencies of existing datasets, that are frequently incomplete, non-standardised, retrospective, non-accessible, siloed and local [44-46]. The concept of a global or even pan European pan-cancer registry, focussed on NGS testing, treatment selection and outcome, would provide a large and high-quality data set and could offer valuable, much needed insights to help chart more personalised clinical pathways and also provide valuable learning on what is needed to share data across borders. The appropriate governance framework would need to be addressed but could build on initiatives such as Million European Genome initiatives as well as the European Health Data Space. Building such a large data set that links diagnosis including NGS and biomarker testing, treatment and outcome data creates a new realm of opportunities, not just in regards to the insights generated by the data itself but also in regards to taking learnings from the challenges of setting up such an initiative. These insights are important on many levels, they will help identify critical data variables and point to important data standards and demonstrate where local regulations prevent or make it difficult to share data across borders. In the context of data insights, valuable information pointing to current use of NGS technology, its impact on clinical decision-making and ultimately impact on patient outcomes is equally important as well providing insights on how treatments were accessed by patients, outcomes, in terms of real-world overall survival and progression-free survival, and best clinical response. This could serve discussions of the use of real-world data to increase the level of knowledge on personalised healthcare and support patient access to NGS testing and innovative therapies [47-49].

Point of care for these ambitious plans is that the data should come from standardised laboratories where samples are collected, transported, stabilised and stored under standardised conditions and include sample metadata on the complete preanalytical phase. This way, the data can be compared and analysed as it is not polluted with irreproducible and or unusable data for further analysis. The quality system ISO 15189 needs to be implemented in these laboratories and complemented with the newly developed CEN and ISO pre-analytical standards that recently have been [50-63] published to ensure the data is comparable with results that are better reproducible.

The health cluster of Horizon Europe should prioritise research to allow better identification/discrimination between significant and insignificant cancers and in areas of personalised cancer medicine. This should allow better identification of patients who will benefit from a particular treatment, thus minimising costs for non-justified use of novel therapy agents, and would delay onset of tumour progression on the basis of appropriate knowledge on biomarkers and validated targets. In order to be able to achieve that goal, integrative projects with novel models, bioinformatics analyses and advanced technologies should be supported [64].

\section{Lessons from the Case Studies}

There are some important generalisations that arise from these case studies. Biomarkers can help to diagnose disease, predict and guide treatment decisions, including determining eligibility for targeted treatments. Advanced testing can provide more accurate or sensitive additional information, widening testing options from protein-based to genetic, and from single gene to NGS [2]. Data generated from the -omics allow the rapid identification and quantification of thousands of molecules in a single process. Multi-gene testing can prognose 
Fig. 10. Tackling the unmet needs in access to biomarkers testing.

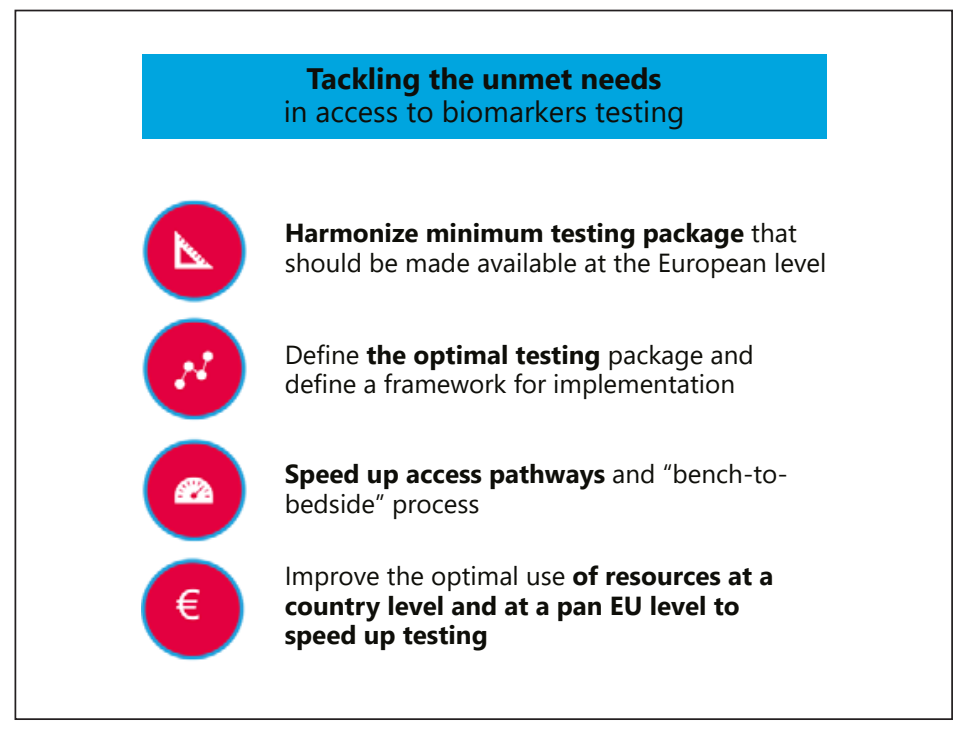

outcomes and can also mitigate risk in families in cases of ovarian cancer: knowledge of BRCA status can inform decisions about mitigating a person's own risk, and support identification of at-risk relatives [1].

Sampling is an issue. Some testing (gBRCA) can be conducted using blood or saliva, but others require tumour tissue. This is the case for tBRCA in ovarian carcinoma or for PIK3CA in breast cancer, and frequently for biopsies from metastatic disease in NSCLC, which is obtained - not without difficulty - with a needle, producing only small (and sometimes poorly informative) amounts of tissue (and with the risk that single site biopsy may not reflect the entirety of genetic lesions in other metastatic sites). Plasma testing (liquid biopsy) can be used in some scenarios for NSCLC (e.g., for EGFR mutation testing) or breast cancer (PIK3CA mutation testing). There is a need to assess the best tissue type to obtain the best information, for example by using a plasma ctDNA test or run both in parallel when a patient only has a small tissue sample, to maximise the chance of a having all relevant biomarker information available prior to treatment decisions being made.

Single hotspot mutation gene testing, still widely used, also has disadvantages. Where plasma testing cannot be used, limited quantities of biopsy material present a problem when multiple individual tests have to be conducted, and the optimal technology is not consistent across the single biomarkers [65] (Fig. 10).

Collaboration has to be systemised to provide for the engagement of multiple specialties in shifting testing objectives from risk assessment to informing treatment decisions. There are specific obstacles that must be overcome if unmet needs are to be satisfied through greater access to biomarker testing. Agreement would be needed on a harmonised package of testing opportunities that should be available as a minimum across Europe. An optimal testing package would also need an agreed definition. Access pathways would have to be speeded up to ensure that valuable innovations became integrated into health systems. And to speed up testing itself, the right resources would need to be deployed in the right way at national and European level.

Timing is also vital for early diagnosis, and efforts are needed to speed up all aspects of the process: BRCA testing, for instance, has historically been delivered through clinical genetics, with a timeframe not geared to informing treatment decisions. Timing is also linked to capacity for handling the volume of tests needed, which usually grows continuously following the introduction of targeted treatments. There is a particular need to overcome the problem of timing in NGS studies. 
Table 7. Recommendations healthcare system - stakeholders recommendations

- Healthcare systems across Europe to create a systematic reference framework for clinical laboratories.

- $\quad$ Regulators, HTA evaluators and payers to align on standardised outcome measures, systematic data collection, and data standards and sharing.

- Developers of biomarker tests to support applications with robust data.

- Developers of biomarker tests, PAGs, HCPs medical societies to cooperate on information and education.

- Drug and diagnostic developers, clinicians, biologists, biostatisticians and digital technology groups to cooperate on pan-cancer studies.

- Laboratories to obtain the general medical laboratory standards ISO 15189 or equivalent accreditation and undertake a minimum of 100 testing per month.

- Laboratories to obtain accreditation on the pre-analytical ISO/CEN standards.

- Central biobank facility at each medical academic centre collecting biomaterials from the existing diagnostic pathways for research accredited for the general biobank standard ISO 20387 and the pre-analytical ISO CEN standards.

It is necessary to determine the optimal sequencing of tests, for instance so that BRCA tumour and germline tests can support fully informed treatment decisions for the greatest number of patients - and to meet different needs for consent and counselling. The choice and sequencing of tests also has implications for tissue acquisition - for instance where it requires involvement of clinicians, surgeons and pathologists.

The uncertainty around commissioning and reimbursement is repeatedly reflected in discussions about testing needs across a range of tumour types, including when determining eligibility for some targeted treatments reimbursement is an issue. Coverage demands remain long and difficult to fulfil for novel biomarkers. There are no guarantees for manufacturers of the adequate reimbursement of tests, and reimbursement requests could take many years before a public (or private) reimbursement is issued. Determining the value of information brought by testing is the primary challenge to the existing reimbursement mechanisms. Predictive biomarker testing should only be performed in close context to clinical treatment decision, i.e. in multidisciplinary tumour boards, so as to avoid testing that is unnecessary and not clinically informed. But access to testing is also inhibited by the lack of awareness of genomic testing possibilities among key stakeholders, and by evidence requirements for the adoption of tests that set disproportionately demanding criteria [66].

There are complexities still to be resolved - such as the impact on demand for testing as a consequence of approval of targeted treatments in new indications, the introduction of new tests in the same indications to identify different sub-groups of patients, or the interplay between risk assessment and treatment decision-making [67].

And there are opportunities still to be explored - such as providing simpler testing kits for complex conditions, and notably the development of blood biopsy, or the convergence of complex test offerings with predictive protein-, genetic-, epigenetic-based biomarkers or NGS panels, or the development of predictive potential of prognostic tests (effectively as companion diagnostics) with emerging drugs.

All types of data are in need of urgent improvement. Agreement is needed on the metrics needed for clinical outcome measurements, for defining the evidence framework, for generating the evidence to support the wide range of stakeholders - clinicians, researchers, payers, policymakers. The better capture of data at different stages in the treatment pathway is a further challenge [68] (Table 7). 
Horgan et al.: Bringing Greater Accuracy to Europe's Healthcare Systems:

The Unexploited Potential of Biomarker Testing in Oncology

\section{Wider Lessons}

The opportunities and challenges illustrated by these case studies on cancer are replicated across the wider field of healthcare - and their enumeration here should be a spur to reflection among policymakers as to how the opportunities can be leveraged.

\section{Insufficient Access}

Molecular diagnostics are not yet well embedded into clinical practice in Europe, so access remains unequal across countries and sometimes even within individual countries, where regional or even local health systems are in place. In some cases, regulatory environments are blamed: advocates of multi-gene expression profiling in breast and prostate cancer complain of an "unrealistic evidentiary burden, including requirements for long-term prospective studies analogous to therapeutic trials" [7].

Access in terms of adoption and uptake is also governed by local regulations: in some countries, only testing for clinically actionable mutations is available, and not broader NGS panels. Reimbursement and value assessment also remain major hurdles, as the responsible national and regional authorities often reach divergent decisions, sometimes with unrealistic desires for demonstration of immediate cost savings. Many payers expect diagnostics to be at least cost-neutral and are often resistant to appreciating the value in terms of information that these technologies may bring to clinical decision-making and to healthcare systems efficiencies. HTA and payer evaluations are more complex and variable, because of the many groups involved. Heterogeneous expectations, resulting from varying national priorities and cultural differences, result in different HTA recommendations on the same intervention. The biggest issue for biomarkers that are assessed via HTA is the non-fit for purpose methodology (often too close to HTA as used for pharmaceuticals). Value of diagnostic information provides an alternative assessment framework beyond conventional cost-effectiveness metrics.

Also, the HTA recommendation is often not linked to a reimbursement decision. Reimbursement decision-making for precision medicine and biomarker testing remains the responsibility for each country. Payers are reluctant to finance new biomarker tests until they are proven to be of value, and also because of costs. Value-based access programs, or "coverage with evidence development" programs can address open questions that payers might have, and at the same time ensure timely access - but they do not exist for IVDs. This can create challenges for labs preparing for new medicine launches, leading to a lag before testing is fully implemented.

The problem is amplified by a lack of reliable evidence, such as robust data, to provide a strong, long-term cost-benefit supporting argument. There is still a lack of centralisation of data in Europe, despite advances made by some countries at national level. In addition, there is no clear funding structure for the implementation of biomarkers into clinical practice. One of the consequences for companion diagnostics is to aggravate the time lag between drug approval and approval for the corresponding test in Eastern European regions [69].

There is insufficient familiarity in health systems with complex testing systems. Reimbursement of biomarker tests that are matched to treatments with positive clinical trial data, or for patients with single mutations, is often easier because of the link to a single medicine. In these cases, it is clear that performing the biomarker test is useful and provides access to treatment that improves a patient's chance for survival and quality of life. The problem arises when health systems need to be able to assess value in more complex testing services, such as testing for biomarkers that look at monitoring disease or patients' health, or that predict whether a patient will have an adverse reaction to a medicine. And even then, reimbursement remains a challenge. 
Horgan et al.: Bringing Greater Accuracy to Europe's Healthcare Systems:

The Unexploited Potential of Biomarker Testing in Oncology

Guidelines related to HTA methodology for companion diagnostics and IVDs often do not account for the specificities of the diagnostic component, and they often include methods very closely related to that used to assess medicines, posing unrealistic and non-fit-for purpose evidence requirements which further impede value demonstration and patient access. The siloed nature of resource allocation within certain healthcare systems also repeatedly delays diagnostics commissioning. In some countries, reimbursement of laboratories will not cover testing for abnormalities that are not part of a hospital's approved formulary, on the grounds that the health service is not responsible for paying for research. Because the generation and implementation of biomarker tests is a multi-step and multistakeholder process, from the initial research for the assay to application into clinical practice, it is particularly vulnerable to siloed budgeting in healthcare systems, which tend to split spending into separate headings for medicines, staff, technology, and so on. In this context, biomarker testing is seen as a grey area for funding, as it sits between research and care for patients - leaving ambiguity over whether it is the responsibility of hospitals, industry or the central health system [70].

The lack of clear funding structures impedes adoption of biomarkers in clinical practice. Diagnostics account for $<2 \%$ of total healthcare spending and influence $60 \%$ of clinical decision making, but the reimbursement policy framework today fails to take this into account and urgently needs updating [71]. What is currently missing for innovative diagnostic technologies is a dedicated and specific reimbursement pathway, on the basis of the value of information delivered, implemented consistently across Europe. Models of well-evolved HTA mechanisms - if connected to a reimbursement decision - would inspire other countries without relevant structures. In such a system, decision makers, including HTA bodies informed by input from patients, would define evidentiary standards for diagnostics and would commit to pay for products that met them. Companion tests approved through the European system would be granted rapid reimbursement, and specific budgetary provision would be made for tests. Public-private cooperation would lead to wide testing availability across the EU in the medium term. The fact that medicines and medtech have two different access pathways (including regulatory approval) is the true challenge [72].

There are also the different forms of value that diagnostic information can provide, depending on the perspective and motivation of each group of stakeholders. For patients, it can offer clinical benefit, empowerment or satisfaction. Healthcare systems stand to gain new efficiencies through patient triage and reduction of health deterioration through disease progression. Healthcare providers can benefit from faster turn-round times of patients, and HCPs can improve patient management through improved clinical response.

Even when a biomarker test has been granted reimbursement, implementation in clinical practice is a further hurdle. Stakeholders must structurally prepare local testing sites, including reviewing capacity, standardised infrastructure, staff training and investments, and discuss where in the patient pathway the testing will take place. Additionally, further testing may be needed once a patient has disease progression to metastatic cancer, as individual metastases may acquire new mutations. Expertise must also be recruited to carry out different aspects of the process related to biomarker testing, such as interpreting biomarker-related genomic data from DNA sequencing in the context of the disease, artificial intelligence, infrastructure management for the logistics of transporting samples for analysis and genetic counsellors. And at all times, the test must meet the technical and quality standards to assure consistent results across all testing sites. Understanding is needed of the infrastructure and quality assessments for appropriate testing to be made available throughout a country, including integration of the test into current treatment algorithms (relating to its place in the complex diagnostic/treatment pathways and multiple lines of therapy for many cancers, for instance). Testing standards and guidelines - on issues such as pre-analytics cut-off thresholds 


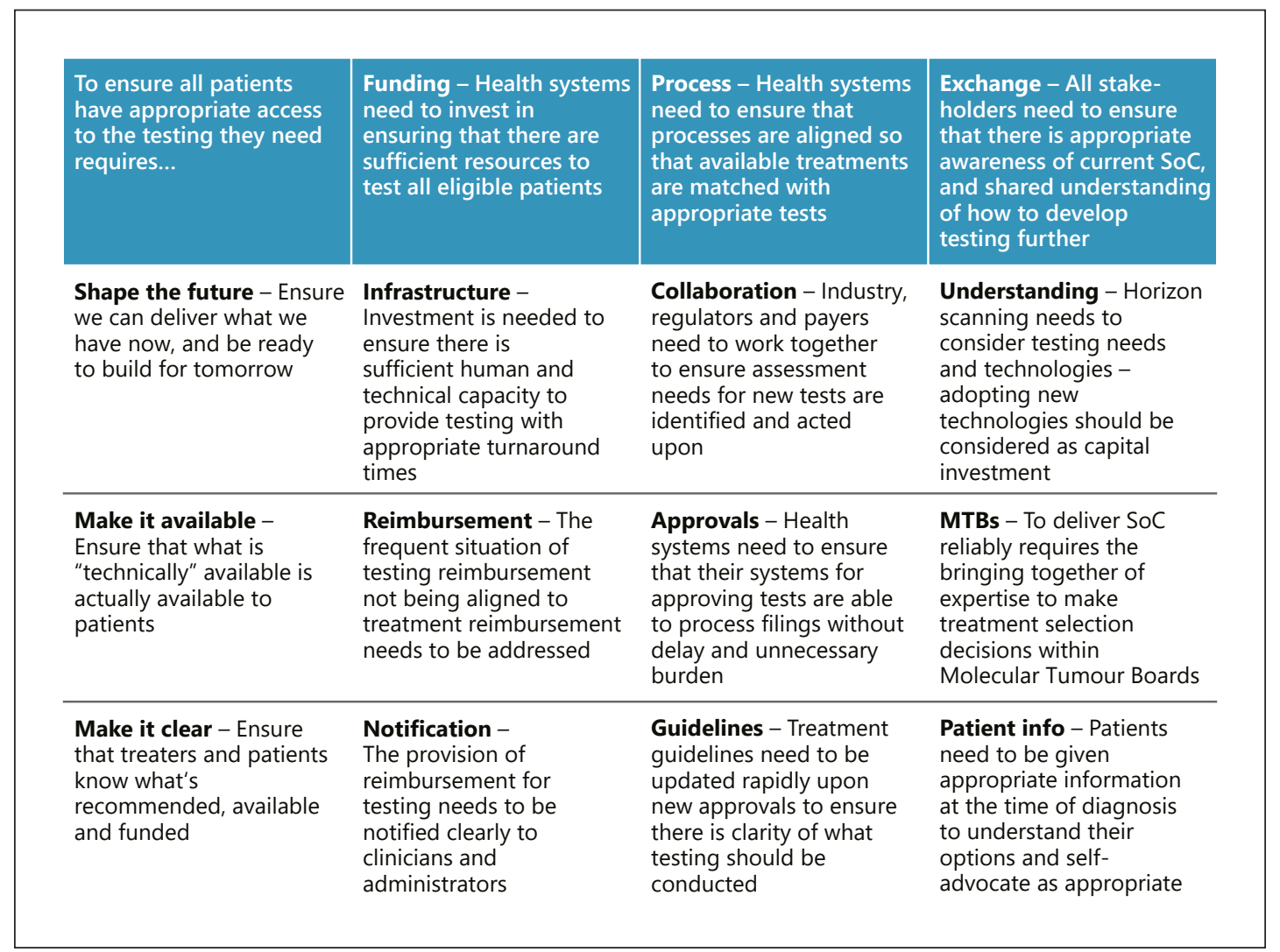

Fig. 11. Multi-stakeholder engagement, pragmatism and focus to secure improvement.

and day-to-day practices - must be determined with laboratories, areas where standardisation and harmonisation are still absent. Quality assurance standards and external assessment mechanisms should be implemented to ensure biomarker test quality across providers with remediation mechanisms for laggards.

Implementation strategies require an understanding of the infrastructure and quality assessments required as they will influence costs. This could result from integrating a biomarker test into current treatment algorithms or providing the necessary expertise to carry it out. Additionally, biomarker tests need to meet technical and quality standards to ensure consistent results. Barriers here include lack of clarity, at the point of reimbursement, on the implementation procedures, and lab and regional variability in testing quality due to a lack of implementation standards on sample and testing quality [14, 73].

Among countries that provide for coverage of potential biomarkers in certain diseases (e.g., breast cancer), some have established pathways. But this is not widespread in Europe. And for other disease areas (e.g., pancreatic cancer), pathways need to be set up, which can cause delays (Fig. 11; Table 8).

\section{Insufficient Alignment}

Some drugs approved for targeted use are not available because patients lack clear access to the related diagnostic biomarkers. Even when a test has been approved, reimbursed and incorporated into clinical guidelines, it can still take up to a year or more for the test to be routinely integrated into clinical practice. (This can mean patients missing out on EMAapproved and nationally reimbursed treatments during that interval [67].) 
Table 8. Recommendations to Member States

- Member states to agree on a federated structure of national databases.

- Member states should ensure that new validated biomarker tests are rapidly made available to patients.

- Member States have incentivized the development and uptake of biomarkers of limited interest to commercial companies (early detection biomarkers \& risk biomarkers).

- Member states/EMA to better synchronise CDx approval processes with drug approval.

- Member states to provide infrastructure for CDx evaluation and demonstration of clinical utility.

- Member states to provide clarity on funding routes for biomarker testing.

- Member states to allocate resources specifically for discovery and validation of biomarkers, and promote engagement between payer organizations, biomarker developers and the wider healthcare stakeholder community (vertical integration and horizontal integration).

- Member States to ensure horizon scanning processes are in place to support early multi-stakeholder engagement on potential challenges for reimbursement and implementation of new tests, and to ensure clear reimbursement structures are in place for biomarker testing.

The advantages of companion diagnostics are well recognised, in principle: EMA's Pharmacogenomics Working Party sees biomarkers will increase in importance in the context of medicine development, and the EMA's revised guideline on the clinical evaluation of anticancer medicinal products offers guidance on regulatory expectations for biomarker-guided development. There is no serious dissent from the view that they provide valuable information, allowing patients, health practitioners and payers to decide with a higher level of certainty about the potential benefits of a treatment or care pathway, with consequent benefits in the efficient selection of treatments and care, targeted at subpopulations that are most likely to benefit [3]. There are also indications of their economic value, even if deficiencies in relevant clinical and economic evidence and the plausibility of assumptions can make the economic case for precision medicine a challenge to demonstrate. Some diagnostics can make a clear case for bringing indirect economies: predictive molecular tests such as the Oncotype DX ${ }^{\circledR}$ test in early stage breast cancer have the potential to pay for themselves by reducing chemotherapy use and cost, but because those costs are borne by different budgets within the healthcare system, no one party has a clear incentive to fund the test to recoup the savings and spare the patient unnecessary chemotherapy. A multi-gene recurrence score assay companion diagnostic test to select breast cancer patients who will not benefit from additional chemotherapy has demonstrated savings in the United States in terms of adjuvant chemotherapy, supportive care, and management of adverse events of USD 1,930 per patient tested. By using KRAS companion diagnostic testing to select KRAS wild-type metastatic colorectal cancer patients suitable for EGFR inhibitor therapy (cetuximab or panitumumab), USD 7,500-12,400 per patient could be saved in the United States and EUR 3,900-9,600 per patient could be saved in Germany with equivalent clinical outcomes. Using KRAS testing would save over EUR 11 million per year in Germany. The consequence is more efficient use of healthcare resources while optimising patient outcomes [74].

But in Europe (in contrast to the US and Japan), the adoption of novel biomarkers and companion diagnostics is far from straightforward. Although they account for a very small proportion of EU spending on in vitro diagnostics, companion diagnostics do not enjoy recognition by health systems of the value they bring to personalised healthcare, and coverage for many companion diagnostic tests varies across Europe, with different time-frames, different value assessments, different criteria, different authorities, and different updating schedules. This inhibits patient access to personalised treatments and care, preventing improved outcomes. 
Their approval is also complicated by the fragmented nature of market access frameworks for diagnostic tests in many European countries, where non-alignment with specific funding and reimbursement mechanisms disincentivises their use. Despite the dependence for success of personalised medicine on the performance of the diagnostic test in terms of its sensitivity, specificity, accuracy and precision, the regulatory approval process in the EU for a diagnostic test and a corresponding medicine remains largely disconnected. The new EU IVDR legislation - still not yet applied - provides a definition for a Dx as a device essential for the safe and effective use of a corresponding medicinal product to identify patients most likely to benefit or likely to be at increased risk of serious adverse reactions. It also establishes a link between the assessment of a CDx by a notified body and the corresponding medicinal product by a medicine regulatory authority or EMA. But more dialogue is needed between medicine authorities, device authorities and notified bodies on establishing the details of the consultation procedure $[75,76]$.

Access to precision medicine is impeded where reimbursement decision-making differs widely between a medicine and a companion diagnostic, and an alignment of the decisionmaking processes merits consideration. The uncertainty on access, funding and uptake of molecular diagnostics, such as companion diagnostics, also has a direct influence on investment decisions. As higher uncertainty leads to lower access to external funding for the development of innovative molecular tests, innovation may be hampered.

The situation in Europe contrasts with the US experience, where the FDA has developed processes for the qualification of biomarkers and other medical product development tools, underscored by legislation, and that permits automatic approval for a biomarker in a single drug development program, relying on direct communication in dialogue between sponsor and reviewers. The result is that companion diagnostics are frequently approved and reimbursed at a suitable level concurrently with therapies developed for the specific patient subpopulations selected [8].

\section{Data Deficiencies}

Advances in genomic sequencing mean that generating genomic data is no longer a major barrier, and the main challenge is being able to interpret a genomic finding in the context of the individual patient. That requires the knowledge gained from data at a population level to sort through the "noise" of variation in an individual's genome and to identify an individual change or pattern that may be unique to an individual.

Data collection, data quality, data standards and data interoperability are notoriously deficient in many of Europe's health and research systems - aside from the Netherlands, where testing standards and quality are high because biomarker testing is channelled through certified laboratories with fixed guidelines, strict protocols and standardised assays [77]. However, even there the quality of the sample and standardisation needs to be improved. To improve reproducibility, not only ISO 15189 is important, but should be complemented with standards on the pre-analytical phase. This would increase the data quality and interoperability due to the addition of sample metadata on the complete sample pre-analytical phase.

For biomarker testing, data is the indispensable component of credibility - and as long as data is seen as sub-optimal, any technologies dependent on it will remain marginal. Without clear directives on quality standards for different biomarker tests, the consequences could be inaccuracies in lab reports, or results that may need further clarification. For policy makers, with the volume of biomarker tests that are coming through for reimbursement, presented data may be conflicting, confusing or, from their perspective, potentially biased based on where the information is coming from, and may create confusion on priorities for decisionmaking. 
Variability in testing quality and accuracy of results not only across Europe, but also nationally between labs, causes a lack of confidence in biomarker testing as well as the data acquired from the tests [78].

There is a parallel issue with information. Europe-wide levels vary in background knowledge and literacy on biomarker education, as well as learning motivation, across stakeholders and geographical regions. There is a lack of understanding of the value of the information delivered by biomarker testing due to knowledge gaps across stakeholder groups and unbalanced knowledge across disease areas. When stakeholders are unconvinced of the value of a biomarker test, even if it is available, they are not likely to recommend it, provide reimbursement or ask for it. Patients who may benefit from it, members of multidisciplinary teams who may recommend it, physicians who may prescribe it, laboratories who may provide results from it, and medical societies who will write guidelines about it - they all need clear information about the test's purpose, characteristics and benefits, backed by reliable data. With new technologies being implemented constantly, all types of stakeholders find it difficult to keep pace with what is available, and while there is wide support for the concept of health literacy, it is not always clear who should be providing that information [79].

Striking examples of the perils of inadequate data have been provided by the COVID-19 pandemic. It has highlighted the impotence that results from insufficient data. More collection of data from more testing would have aided understanding of different spikes, and planning of where resources should be allocated, of who to test and how to test, and how to treat and follow up patients more effectively, and what preventative measures should be put in place. It has also revealed gaps in capacity, both in developing and producing test kits, and for expertise in conducting and processing tests at scale and speed. And it has given new currency to discussions of who qualifies for testing - only symptomatic patients, or asymptomatic too? The pandemic is raising awareness among clinicians and driving testing practice. It remains to be seen whether it will lead to more funding and capacity for biomarker testing in general, and to making effective tests more readily available across a broader range of health issues [80].

There is a need for concise, accurate and directed education around biomarker testing to increase comprehension of how the information from biomarker testing impacts individual stakeholder decisions, whether in choosing medicines, creating policies, or defining infrastructure. Barriers include lack of understanding of the value of biomarker testing due to knowledge gaps across stakeholder groups, and lack of resources for stakeholders to access up-to-date information.

\section{Potential Solutions}

A long-overdue update to the European Commission's 2003 recommendation on cancer screening could provide a spur to remedial action across many of these urgent needs. The update should reflect changing times, potential and new knowledge, so as, crucially, to include provision for early diagnosis through molecular diagnostics/biomarker testing [81].

\section{Funding Solutions}

- Healthcare budgets and funding processes should be redefined, with immediate funding clarity over biomarker testing reimbursement processes, specific budget allocations for biomarkers and innovative medicines, and the gradual introduction of new models of reimbursement.

- Decision makers should define the value considerations they seek from diagnostic technologies and should commit to pay for them, with a dedicated value-based reimbursement 
pathway based on the value of information provided, clear evidence requirements, and implemented consistently across Europe.

- Healthcare funding bodies should introduce rapid procedures for reimbursement of biomarker testing at a suitable rate to incentive the use of IVDs.

- Member states should ensure that new biomarker tests are rapidly made available to patients and should be open to dialogue with sponsors during development to address potential challenges.

\section{Data Solutions}

- Biomarker testing should be of high standardised quality on high-quality samples, with defined testing pre-analytical and analytical standards and clear criteria on minimum performance characteristics of biomarker tests.

- Data sets accompanying biomarker tests should be robust and clearly demonstrate a relationship between the biomarker and its expected value.

- Member states should each create a national system to validate and set up tests, and Europe should move towards data centralisation, to permit the creation of robust data sets across the continent to generate cost-benefit evidence. This should include clear minimum criteria and formats for reporting in terms of standardised metrics taking account different stages of testing.

- Data sharing policies across member states should be revisited to facilitate European multi-institutional and multi-national studies every 3 years, with appropriate attention both to the movement of data and relevant privacy legislation.

Europe-wide initiatives already underway for centralising genomic data may provide some models. A repository for haematological malignancies for a 100,000-molecule database is under construction as part of the Innovative Medicines Initiative's "Big Data for Better Outcomes" project, to bring biomarkers back to the clinic [82]. A European Commission project to sequence 1 million + genomes by 2022 has been signed up to by more than 20 countries, and EAPM's MEGA+ initiative aims to facilitate the sharing of all relevant health data, including - but going beyond - genomes [83]. A UK initiative is bringing together 1 million patient years of precision oncology and knowledge to interrogate the data to advance clinical implementation. "Until we learn from every patient, we can never full test the therapeutics we need to test, and we cannot know how this will work and therefore we won't be able to progress at a fast rate," according to its officials. France's Médecine Génomique 2025 also encompasses research and enterprise, and there have been significant long-term investments and strategies relating to genomics in Estonia, Denmark, Iceland, and Finland [84].

\section{Information and Education Solutions}

- Stakeholders should receive tailored biomarker education to ensure appropriate timing and breadth of information, including ethical and compliance considerations.

- The short-term focus should be on educating groups highly influential in widening access to biomarker testing, notably payers.

- Biomarker education should be maintained to keep stakeholders upskilled so that they can make informed decisions.

- Information bearers should be identified at a Europe-wide and member state level, along with support for their role and visibility.

Tailored biomarker education should ensure appropriate timing and breadth of information per stakeholder (i.e., patients and carers, HCPs, policy makers, payers, government officials, etc.), with priority to payers. EMA's "regulation for 2025" urges support for develop-

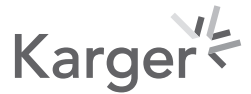


ments in precision medicine, biomarkers and 'omics, and early engagement with novel biomarker developers to facilitate regulatory qualification. It also plans to review its biomarker validation process to encourage greater uptake and use, and to evaluate, in collaboration with HTAs, payers and patients, clinical outcomes measured by biomarkers. It also plans a process for multi-stakeholder scientific advice to support development of medicine-device combinations, qualification methodologies and the use of companion diagnostics [85].

\section{Implementation Solutions}

- Early engagement with regulatory bodies should be routine to address challenges and provide clarity on the infrastructure needed.

- Countries should allocate funding to support this.

- National authorities should pre-emptively organise meetings or courses on how to implement biomarker tests and provide clarity for all stakeholders on the procedures needed to implement a biomarker test, to allow timely access across testing centres.

The cumulative effect of such improvements in the context for biomarkers should, at a future date, also underpin a situation which might be described as optimum testing.

\section{Optimum Testing}

In an optimum biomarker testing scenario, excellent clinical teams all over Europe would deliver a high standard of care for every patient with cancer. Complex multi-modality teams of experts, adequately trained in molecular diagnostics, would be the norm to allow effective decision making as more information and more biomarkers become available. Pan-cancer studies would operate on the basis of standardised data between multiple platforms, with agreed criteria among different groups of researchers to work on the data and present the results. Registries for each disease area would provide more robust data sets - including the patient's disease history, outcomes, treatments and genomic aspects, and information on the use of biomarker tests themselves, such as how often and on which patients [86] (Fig. 12).

Accredited testing facilities for ISO 15189 (or equivalent) complemented with the preanalytical ISO/CEN standards is needed for reproducibility and generation of useful datasets and registries. In addition, scientific medical studies based on biomaterials derived from patients should also use the ISO/CEN pre-analytical standards to collect the samples. This can best be organised in centralised biobank facilities in every academic medical centre, which are for the collection of the biomaterials integrated in the existing diagnostic pathways and accredited for ISO 15189 (or equivalent) and the complementary pre-analytical ISO/CEN standards. This way, the scientists use standardised samples to discover biomarkers which are better reproducible in the validation process needed for the test to become a diagnostic test [14]. The sample metadata describing the complete pre-analytical phase of the sample needs to be included in the publication can be used to analyse and define the precisely needed pre-analytical conditions for a robust test result. Most importantly, pre-analytical conditions not favourable for good test results can be recognised and avoided. In addition, samples that are not reliable for the test can be recognised before they are going to be used and can be rejected.

The complementary pre-analytical ISO/CEN standards published so far describe: DNA, RNA and ccf DNA isolated from venous whole blood, RNA, DNA and proteins isolated from FFPE tissues or frozen tissue DNA isolated from saliva, DNA, RNA isolated from circulating tumour cells (CTCs) from venous whole blood staining of CTCs and metabolomics in urine serum and plasma [55-63]. More documents of the pre-analytics series are expected in the near future.

\section{Karger's}




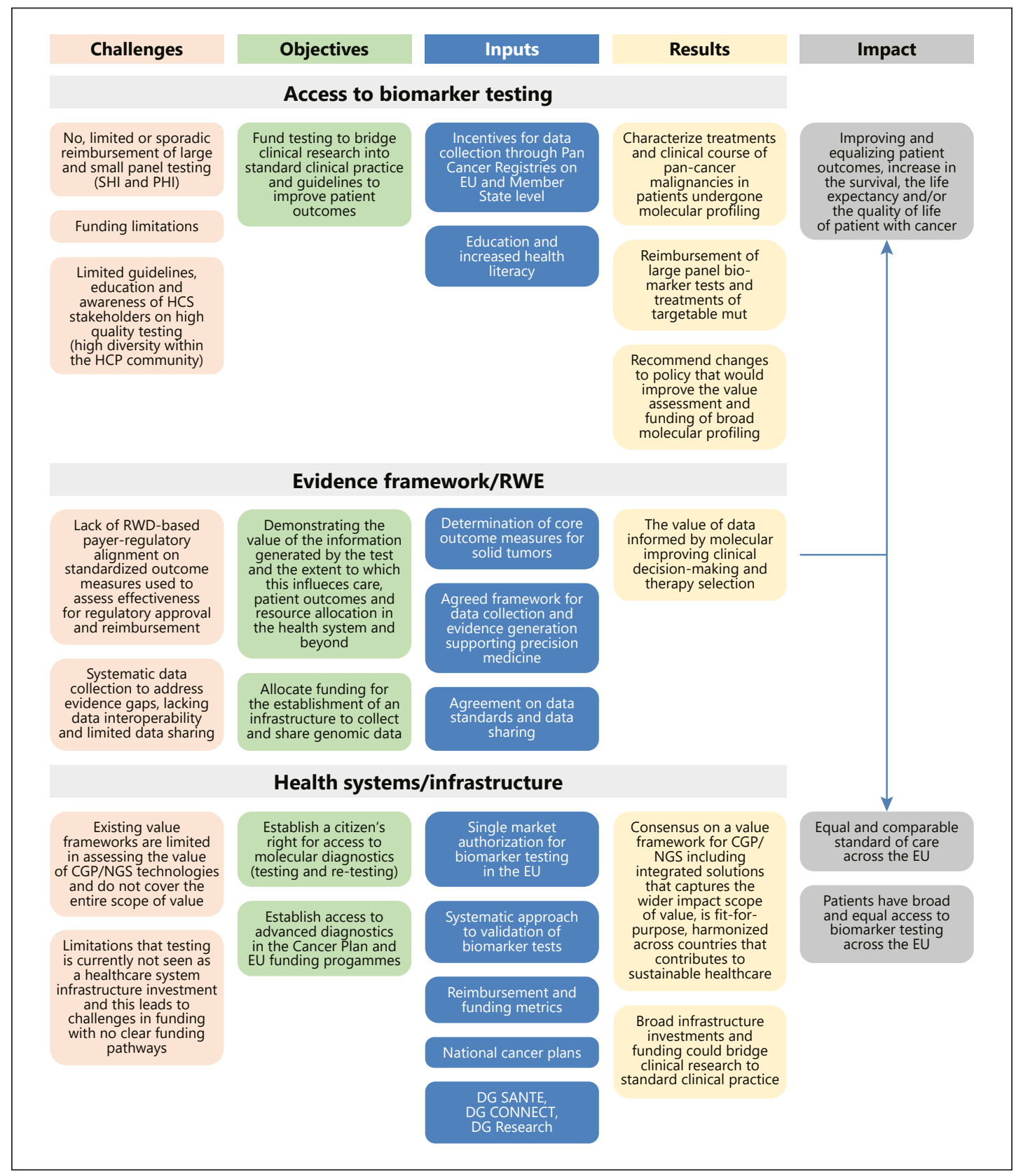

Fig. 12. Optimum testing: challenges, objectives, inputs, results and impact.

This scenario looks expensive and difficult to organise, but the return on investment would feature rapid validation of new biomarkers, reimbursement of testing, quality-assured laboratories in all regions, wide horizon scanning, constant updating of guidance, refined testing algorithms, and fast turnaround times of testing to allow early diagnosis. Early engagement with policy makers, budget holders and assessors during the development of a biomarker test would clarify challenges to reimbursement and implementation, with data on health, economics, patient outcomes and usage demonstrating how the test provides value to the healthcare system. Specifically, for companion diagnostics, clear, transparent and timely market access processes would be put in place in all European health systems, overcoming 
Table 9. Recommendations to EMA

- $\quad$ EMA to progress its plans for early engagement on regulatory qualification of novel biomarkers.

- $\quad$ EMA to review its biomarker clinical and analytical validation process.

- $\quad$ EMA to evaluate clinical outcomes measured by biomarkers.

- $\quad$ EMA to progress multi-stakeholder scientific advice on the use of companion diagnostics.

silo budget structures, and with reimbursement processes recognising the value of diagnostic information, and backed by funding streams and uptake mechanisms [87].

There would be coordination at European level among member states to support better use of molecular diagnostic testing and to adapt healthcare systems accordingly. EMA would ensure that the latest scientific and technological knowledge was built into medicines development to benefit public health. There would be less invasive sampling methods for diagnosis, simpler testing kits for complex conditions based on the development of blood biopsy where appropriate, the convergence of complex test offerings with predictive NGS panels, and the full development and acceptance of the predictive potential of prognostic tests with emerging drugs.

Infrastructure would support large screening platforms to identify target populations, and lifelong monitoring would deliver meaningful information in terms of early diagnosis on risk prediction, molecular and physiological phenotyping and treatment outcomes. Regulatory procedures across Europe would be simplified, aligned and predictable. Dialogue would be routine among decision-makers and manufacturers from early in development. Regulatory pathways would be adapted to promote efficient development of new diagnostics and therapies, and evaluation pathways adapted to promote efficient and rapid uptake [88, 89].

Early engagement would also allow stakeholders to structurally prepare local testing sites, ensure capacity, infrastructure and investments, and discuss where in the patient pathway the testing will take place. It would allow laboratories to determine any testing standards and guidelines for each biomarker, such as quality assurances, cut-off thresholds and day-to-day practices, to provide for equal access to the same testing (Table 9).

\section{Exploiting Genomics to the Full: The Need for a Policy Framework}

A policy framework for biomarkers in the EU would guide member states and facilitate consistent decision making, allowing molecular diagnostics and biomarkers to be integrated into the EU's divergent healthcare systems. The EU Cancer Plan would establish access to advanced diagnostics, with appropriate funding, providing all citizens with the right to molecular diagnostics. All relevant factors for each patient would be fed into a personalised probability distribution for any given outcome. The clinician could combine elements of evidence from different sources, from molecular biology to randomised trials, and adjust probabilities of a treatment's benefit to the single patient, rather than just to an average. This would make it possible to view the patient's profile in the context of other patients also susceptible to treatment. And by making these factors explicit, the chances would be raised of improving care, by permitting comparison of assumptions as well as outputs [4].

Fast and effective sequencing technology coupled with reduced costs would enable increasing volumes of data to be assimilated on individual patients and their diseases, preand post-treatment. The scope of the molecular information that will be available to clinical researchers, and eventually treating physicians, would expand dramatically, and this detailed information would make it possible to tailor medical interventions, including combinations, 
to individual patients at first diagnosis and throughout the course of their disease as potentially new variants arise and response to treatment diminishes.

Advantage would be taken of genomic data's potential to match the genomic profile with clinical data generated with similar or overlapping profiles and predict potential outcomes. Routine use of comprehensive genomic sequencing, computational power and advances in bioinformatics would allow the analysis of complex genome datasets in real time, generating a dynamic pool of curated genomic and clinical data, drawn from multiple sources. New applications of NGS, e.g. RNA sequencing, would enable the analysis of gene expression in tumour samples supply additional information to DNA sequencing. This, together with, for example, proteomic approaches will add further insights into the functional role of genomic alterations. Some applications combining testing using NGS with artificial intelligence and machine learning may ultimately have the potential to help chart entire clinical pathways [17]. Artificial Intelligence may provide advice for a given specific patient to the clinician, based on the analysis and processing of a multitude of data of a multitude of similar patients. That will require broad multi-institutional and international efforts throughout the EU to feed the emerging AI-based algorithms with well-curated data [90].

Extensive molecular testing would overcome the limitations of serially applied single biomarker tests and circumvent the limitations of tumour biopsy size and availability. Longitudinal sequencing of circulating tumour DNA from ccfDNA would alert to emerging resistance and permit the optimal combination or sequence of treatments.

A paradigm shift could result from the understanding that genomic instability and intratumour heterogeneity drive disease evolution and the dynamic nature of the disease over time. It could be driven by growing portfolios of targeted agents with well-characterised predictive molecular markers, and by comprehensive sampling of tumour DNA (including ccfDNA) during the disease course. Molecular characterisation, in combination with novel treatment approaches including immunotherapies and antibody drug conjugates, would lead to significant improvements in patient outcomes [91].

\section{Acceptance of the Economic Case}

The necessary framework for real-world evidence would be supplied by alignment of regulators and - as well as patient representatives, HPCs and industry in multi-stakeholder alignment - on standardised outcome measures, systematic data collection, and agreement on data standards and sharing [92]. That way, the value of the information generated by the test could be demonstrated, and could influence care, patient outcomes and resource allocation. The value of data informed by molecular diagnostics would be recognised, improving clinical decision-making and therapy selection. The EU would also have to put in place single market authorisation for biomarker testing, alongside a systemic approach to validation of biomarker tests, reimbursement and funding metrics $[93,94]$.

New pricing and reimbursement models would reward innovation and value specific to diagnostics, recognising the value of the information, including in bringing greater certainty in treatment decision making, and adopting a cross-silo approach to healthcare spending. Better alignment between financial incentives and the comprehensive value of new molecular tests would allow more equitable access to these new tests [87].

Fuller embrace of advanced biomarker testing would demonstrate how precision medicine could improve the relative cost effectiveness of care by exploiting patient level heterogeneity in cost and outcomes. Mechanisms that reveal previously unobserved inter-patient heterogeneity via advanced testing would increase the accuracy of predictions of outcomes [95].

Recognition would multiply the cost-effective use of healthcare resources by accurately targeted treatment, improved population health outcomes and reduce the quantity of adverse drug reactions (and the associated cost of their management) [96].

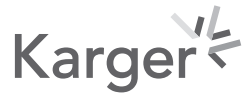


Horgan et al.: Bringing Greater Accuracy to Europe's Healthcare Systems:

The Unexploited Potential of Biomarker Testing in Oncology

Integration of evaluation and reimbursement models would avoid variable and disconnected assessment of supporting evidence for the medicine and its companion diagnostic.

Knowledge of biomarkers is already driving progress toward broader tests that include dozens or hundreds of genetic variants, depending on sophisticated algorithms for interpretation and use. Consensus would be reached among the stakeholders on robust procedures and measures of value, with aligned reimbursement processes for precision mechanisms and subsequent treatment, based on transparent expectations for evidence requirements and study designs adequately matched to smaller target patient populations. And aligned processes for the constituent components of precision medicine would remove uncertainty in the potential for return on investment.

The value of biomarker testing in providing information that can rule out disease risks or the need for certain downstream tests or treatments would be accepted by HTA agencies, and the value perspectives for HCPs, patients and health systems would be recognised. Expansion toward broader testing approaches (e.g., whole genome sequencing) will deliver still more information to broaden the options for ruling out conditions or therapies - which would also be accounted for in value assessment. Data from the leveraging of RWE would also increase the recognition of the merits of biomarker testing [74].

\section{Responding to Opportunities from Advanced Medicine}

Benefits for patients and PAGs would emerge in earlier diagnosis, better care, and assistance in decisions regarding life choices, with improved outcomes in survival, life expectancy and quality of life. Benefits for HCPs (including pathologists, oncologists and researchers) would include access to deeper analysis of a disease so as to differentiate the diagnosis, and to provide direction on the application of specific treatments. Innovation would be brought into healthcare systems faster with real-world data structured to provide the evidence for decisions. Benefits for payers would include a systematic reference framework for clinical laboratories to support market selection of accurate and reproducible tests, providing greater confidence. And with economic evaluation of precision medicine becoming an iterative process with constant generation of new evidence, rather than a one-time standalone activity, it would allow more refined estimates of the impact on patients' long-term cost and health outcomes of using a diagnostic test in clinical practice [91]. Benefits for policy makers would include the potential to identify and reduce disease risk through prevention, early detection and treatment identification, as well as to monitor the effectiveness and choice of treatments, with full support from real-world data. Wide access to quality biomarker testing for early identification of cancer patients, with earlier treatment and consequently better outcomes, would bring cost savings for the health-care system.

Gene therapy is being developed for subtypes of inherited retinal diseases which will require an accurate diagnosis. An NGS gene panel test has been developed to conduct diagnosis at this level of sensitivity, superseding the single and time-consuming Sanger sequence test for each suspected gene. The diagnostic information from the panel test will also reveal the mutation status of patients who are eligible for targeted gene therapy.

The development of liquid detection technologies that use blood or other bodily fluid samples rather than tissue will permit rapid identification of biomarkers with increasing affordability, allowing more precise treatment monitoring.

Precision medicines targeting multiple markers in a single treatment are also beginning to evolve, with the potential to replace existing therapies or combinations. As disease progresses, the process behind these treatments may ultimately enable iterative development of a truly individualised therapy customised to disease progression, improving effectiveness to the point of transformative disease knockdown or cure effect $[97,98]$.

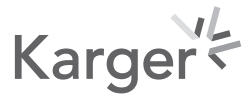




\section{Conclusion: A Seismic Shift in Healthcare Strategy}

Increased molecular understanding is transforming the prospects for healthcare, and molecular diagnostics are opening the pathway to a shift in healthcare strategy. Molecular testing has informed some major preventative public health strategies - such as the significant reduction in cancer mortality. This is the threshold of a new future in which emphasis will shift away from treating illness and move toward maintaining the health of the individual. Early diagnosis and personalised healthcare will have a central part to play, particularly in the cancer arena. The objective for all stakeholders should be to see discovery "hits" translated into robust but affordable clinical "wins" for patients.

The EU is itself in constant evolution, both organically, as its competences are progressively refined, and in response to changes in the world it inhabits. In healthcare, its evolution is marked not only by the emergency of the COVID-19 pandemic, which has largely monopolised the attention of all the major EU institutions throughout most of the early months of the year, but also by the constant increase in morbidity, which now affects more than its ageing population. 2020 was already designated as the year for action against cancer, with two parallel initiatives, and for the initiation of a European Health Data Space and a new Pharmaceutical Strategy. It is an appropriate moment also for it to review its approach to testing as an intrinsic element in an integrated health strategy. In these straitened circumstances, a reappraisal of the significance and potential of biomarkers can provide the EU and its citizens with a much-needed immediate up-tick in quality and accuracy of care, and a change in policy that holds out the prospect of a radical transformation of care in coming years as the full benefits of an optimum approach to the use of biomarkers begin to be felt.

\section{Recommendations}

\section{Recommendations to the EU}

- The European Commission to update by 2021 its recommendation on early detection strategies allowing for risk stratification through molecular diagnostics/biomarker testing.

- European health authorities to put in place a policy framework to support diagnostics in the EU by 2022, with a ring-fenced budget allowance for biomarker testing development (clinical validation). Research on biomarker discovery and early testing should also be a matter of concern and funding-promoting policies.

- The EU to agree by 2023 a business model for public-private cooperation for optimal biomarker testing available across the EU.

- The EU to provide guidance on minimal testing standards and resource allocation by 2025.

- The EU to establish a framework for quality of testing and value of diagnostics information, with a fast track approval mechanism for biomarker validation.

- The EU to facilitate centralised and standardised registries of diagnosis including sequence and biomarker data, pre- analytical sample metadata, treatment and outcome.

- The European Commission should consider a 12-month postponement of the date of application of the IVDR, to mitigate capacity challenges within the notified bodies and the effect of COVID-19 on European stakeholder's preparations for this new regulation. This would be in line with a recent decision for a 12-month postponement of the MDR.

- The European Commission's Medical Devices Coordination Group and the member states' National Competent Authorities for IVDs should develop clear guidance for public and private laboratories on how they should move forward with the development of labdeveloped tests under the new regulation. 
Horgan et al.: Bringing Greater Accuracy to Europe's Healthcare Systems:

The Unexploited Potential of Biomarker Testing in Oncology

Recommendations to EMA

- EMA to progress its plans for early engagement on regulatory qualification of novel biomarkers.

- EMA to review its biomarker clinical and analytical validation process.

- $\quad$ EMA to evaluate clinical outcomes measured by biomarkers.

- EMA to progress multi-stakeholder scientific advice on the use of companion diagnostics.

\section{Recommendations to Member States}

- Member states to agree on a federated structure of national databases.

- Member states should ensure that new validated biomarker tests are rapidly made available to patients.

- Member States to incentivise the development and uptake of biomarkers of limited interest to commercial companies (early detection biomarkers and risk biomarkers).

- Member states/EMA to better synchronise CDx approval processes with drug approval.

- Member states to provide clarity on funding routes for biomarker testing.

- Member states to allocate resources specifically for discovery and validation of biomarkers, and promote engagement between payer organisations, biomarker developers and the wider healthcare stakeholder community (vertical integration and horizontal integration).

- Member states to ensure horizon scanning processes are in place to support early multistakeholder engagement on potential challenges for reimbursement and implementation of new tests, and to ensure clear reimbursement structures are in place for biomarker testing.

\section{Healthcare System - Stakeholders Recommendations}

- Healthcare systems across Europe to create a systematic reference framework for clinical laboratories.

- Regulators and payers to align on standardised outcome measures, systematic data collection, and data standards and sharing.

- Developers of biomarkers to support applications with robust data.

- Developers of biomarkers, PAGs, HCPs medical societies to cooperate on information and education.

- Drug and diagnostic developers, clinicians, biologists, biostatisticians and digital technology groups to cooperate on pan-cancer studies.

- Laboratories to obtain the general medical laboratory standards ISO 15189 or equivalent accreditation and undertake a minimum of 100 tests per month.

- Laboratories to obtain accreditation on the pre-analytical ISO/CEN standards.

- Central biobank facility at each medical academic centre collecting biomaterials from the existing diagnostic pathways for research accredited for the general biobank standard ISO 20387 and the pre-analytical ISO CEN standards.

\section{Data Solutions}

- Biomarker testing should be of high quality, with defined testing pre-analytical and analytical standards and clear criteria on minimum performance characteristics of biomarker tests.

- Data sets accompanying biomarker tests should be robust and clearly demonstrate a relationship between the biomarker and its expected value.

- Member states should each create a centralised national system aligned with European standards to validate and set up tests, and Europe should move towards data centralisation, to permit the creation of robust data sets across the continent to generate cost- 
benefit evidence. This should include clear minimum criteria and formats for reporting in terms of standardised metrics taking account different stages of testing.

- Data sharing policies across member states should be revisited to facilitate European multi-institutional and multi-national studies every 3 years, with appropriate attention both to the movement of data and relevant privacy legislation.

\section{Information and Education Solutions}

- Stakeholders should receive tailored biomarker education to ensure appropriate timing and breadth of information, including ethical and compliance considerations.

- The short-term focus should be on educating groups highly influential in widening access to biomarker testing, notably payers.

- Biomarker education should be maintained to keep stakeholders upskilled so that they can make informed decisions.

- Information bearers should be identified at a Europe-wide and member state level, along with support for their role and visibility.

\section{Implementation Solutions}

- Early engagement with regulatory bodies should be routine to address challenges and provide clarity on the infrastructure needed.

- Authorities should pre-emptively organise meetings or courses on how to implement biomarker tests.

- Authorities should provide clarity for all stakeholders on the procedures needed to implement a biomarker test, to allow timely access across testing centres.

The last decade has seen a remarkable technological explosion resulting in an entirely new field designated as "omics." The application of high-throughput techniques for profiling DNA, RNA and protein in breast cancer samples from hundreds of patients has profoundly increased our knowledge of breast cancer. However, many gaps in our knowledge remain, and these will require a long process of extended clinical correlation studies, deeper integrated "omic" analysis and functional annotation to fill. The major issue that arose during the web consensus is the increasing gap between what is theoretically feasible in patient stratification (genomic testing, NGS approach, disease segmentation) and treatment (dual targeting, integration of targeted agents) and the daily practice. Can we really transfer into daily clinical practice the huge amount of "omics" data? This is a major concern shared by all oncologists. Access to innovation for patients is the issue: how to speed it up? The same limitations are related to clinical practice in the case of expensive drugs to be integrated in patient management (dual targeting in HER2-positive breast cancer). We really believe this is a major political issue: How to create access to innovation for citizens, bridging the gap between clinical trials and daily practice? In many of European countries, many innovative therapies or diagnostic tests are not yet approved or reimbursed. We need to find a new path to access innovation that would translate clinicalresearch into daily practice. Potential solutions for further improvement must entail new biology-driven approaches, since optimisation of conventional treatments has in many cases reached its limits. The introduction of drugs that are less toxic, more targeted and more costly than those currently used necessitates a partnership between clinical and translational researchers, the pharmaceutical industry, drug regulators and patients and their families. This therapeutic alliance will ensure that efforts are focused on the unmet clinical needs of patients with breast cancer. The immediate priority for patients with cancer is to improve access to an affordable, best standard of care in every country. A strong link between quality of care and research activity is dependent on national resources and health-care priorities being aligned to support the improvement of care for patients with breast cancer. To

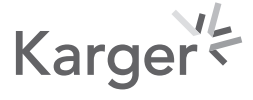


ensure continued innovation that meets the needs of patients, the therapeutic alliance between patients and academic-led research needs to be extended to include relevant pharmaceutical companies and drug regulators. The other key issue around national planning is the increasing complexity of managing the rare and heterogeneous types of cancers with the increasing number of drugs specific for subpopulations. This difficulty creates the need for specialised centres of excellence and referral pathways as important components of care planning, increasing networking in national and continental healthcare programs. For access to innovation for cancer patients, we need to bring together major players from the world of breast cancer research to map out a coordinated strategy, on an international scale, to address this fragmentation. Many programs and investigators are pursuing their own separate paths in relative isolation and generating data without establishing how best to integrate their data with the larger research community. We need a coordinated, collaborative, communal effort to improve access to an affordable, best standard of care for every patient in every country.

\section{Acknowledgements}

We would like to thank the members of the European Alliance for Personalised Medicine, European representatives of the Commission and Member States for their kind input.

\section{Statement of Ethics}

This work was prepared and approved by a multi-stakeholder group of authors which included amongst other representatives from patients' organisations and scientific societies.

\section{Conflict of Interest Statement}

Benjamin Horbach is a Roche employee and has stock options in Roche. Stephen Hall is a Novartis employee and has stock options in Novartis. Benjamin Gannon is a Myriad Genetics employee and has stock options in Myriad. Rocio Varea Menendez is Eli Lilly employee and has stock options in Eli Lilly. Sarah Mee is an AstraZeneca employee and has stock options in AstraZeneca. Susana Banerjee receives grants from Astrazeneca, GSK \& Honoraria - Astrazeneca/ MSD, Amgen, Clovis, GSK, Mersana, Seattle Genetics, Merck Sereno, Genmab, Roche, Immunogen.

\section{Author Contributions}

The work was coordinated by Denis Horgan, EAPM Executive Director and all authors contributed equally to conceiving, building and drafting the article with critical input from all co-authors and experts from within the membership of the European Alliance for Personalised Medicine

\section{Funding Sources}

This work was supported by the in-kind support of the partners of the European Alliance for Personalised Medicine as well as financial support from F. Hoffmann-La Roche AG, Novartis, AstraZeneca, Eli Lilly and Company, Myriad Genetics.

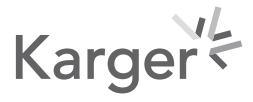




\section{References}

1 Mayeux R. Biomarkers: potential uses and limitations. NeuroRx. 2004 Apr;1(2):182-8.

2 Li AL, Song J. Biomarkers classification and their application in clinical medicine. Zhongguo Yaolixue Yu Dulixue Zazhi. 2015;29:7-13.

3 European Medicines Agency. Committee for Human Medicinal Products. Concept paper on predictive biomarker-based assay development in the context of drug development and lifecycle. 2017 July 20. Cited June 8, 2020. Available from: https://www.ema.europa.eu/en/documents/scientific-guideline/concept-paperpredictive-biomarker-based-assay-development-context-drug-development-lifecycle_en.pdf

4 Berns A, Ringborg U, Eggermont A, Baumann M, Calvo F, Eggert A, et al. Towards a Cancer Mission in Horizon Europe. Mol Oncol. 2019 Nov; 13(11):2301-4.

5 Communication from the Commission to the European Parliament. the Council, the European Economic and Social Committee and the Committee of the Regions, on enabling the digital transformation of health and care in the Digital Single Market; empowering citizens and building a healthier society, $\operatorname{COM}(2018) 233$ final. Cited June 8, 2020. Available from: https://eur-lex.europa.eu/legal-content/EN/TXT/HTML/?uri=CELEX: 52018DC0233\&from=EN

6 European Commission. Roadmap Pharmaceutical Strategy - Timely patient access to affordable medicines, Ref. Ares(2020)2858413 - 03/06/2020 Cited June 8, 2020. Available from: https://ec.europa.eu/info/law/ better-regulation/have-your-say/initiatives/12421-Pharmaceutical-Strategy-Timely-patient-access-toaffordable-medicines

7 Ritzhaupt A, Hayes I, Ehmann F. Implementing the EU in vitro diagnostic regulation - a European regulatory perspective on companion diagnostics. Expert Rev Mol Diagn. 2020 Jun;20(6):565-7.

8 US FDA. Guidance for industry and food and drug administration staff. In Vitro Companion Diagnostic Devices. Cited June 8, 2020. Available from: http://www.fda.gov/downloads/MedicalDevices/DeviceRegulationandGuidance/GuidanceDocuments/UCM262327.pdf

9 OECD, Policy Issues for the Development and Use of Biomarkers in Health. Cited June 8, 2020. Available from: https://www.oecd.org/health/biotech/49023036.pdf

10 Wurcel V, Cicchetti A, Garrison L, Kip MM, Koffijberg H, Kolbe A, et al. The Value of Diagnostic Information in Personalised Healthcare: A Comprehensive Concept to Facilitate Bringing This Technology into Healthcare Systems. Public Health Genomics. 2019;22(1-2):8-15.

11 Khleif SN, Doroshow JH, Hait WN; AACR-FDA-NCI Cancer Biomarkers Collaborative. AACR-FDA-NCI Cancer Biomarkers Collaborative consensus report: advancing the use of biomarkers in cancer drug development. Clin Cancer Res. 2010 Jul;16(13):3299-318.

12 Selleck MJ, Senthil M, Wall NR. Making Meaningful Clinical Use of Biomarkers. Biomark Insights. 2017 Jun;12: 1177271917715236

13 Parkinson RD, Johnson BE, Sledge WG. Making Personalized Cancer Medicine a Reality: Challenges and Opportunities in the Development of Biomarkers and Companion Diagnostics. Clin Cancer Res. 2012 Feb 1;18(3): 619-24.

14 Riegman PH, Becker KF, Zatloukal K, Pazzagli M, Schröder U, Oelmuller U. How standardization of the preanalytical phase of both research and diagnostic biomaterials can increase reproducibility of biomedical research and diagnostics. N Biotechnol. 2019 Nov;53:35-40.

15 Rodriguez BJ, Córdoba GD, Aranda AG, Álvarez M, Vicioso L, Pérez CL, et al. Detection of TP53 and PIK3CA Mutations in Circulating Tumor DNA Using Next-Generation Sequencing in the Screening Process for Early Breast Cancer Diagnosis. J Clin Med. 2019 Aug;8(8):1183.

16 Blok EJ, Bastiaannet E, van den Hout WB, Liefers GJ, Smit VT, Kroep JR, et al. Systematic review of the clinical and economic value of gene expression profiles for invasive early breast cancer available in Europe. Cancer Treat Rev. 2018 Jan;62:74-90.

17 Kamps R, Brandão RD, Bosch BJ, Paulussen AD, Xanthoulea S, Blok MJ, et al. Next-generation sequencing in oncology: genetic diagnosis, risk prediction and cancer classification. Int J Mol Sci. 2017 Jan;18(2):308.

18 Nicolini A, Ferrari P, Duffy MJ. Prognostic and predictive biomarkers in breast cancer: Past, present and future. Semin Cancer Biol. 2018 Oct;52(Pt 1):56-73.

19 Li A, Schleicher SM, Andre F, Mitri ZI. Genomic Alteration in Metastatic Breast Cancer and Its Treatment. Am Soc Clin Oncol Educ Book. 2020 Mar;40(40):1-14.

20 Duffy MJ, Harbeck N, Nap M, Molina R, Nicolini A, Senkus E, et al. Clinical use of biomarkers in breast cancer: Updated guidelines from the European Group on Tumor Markers (EGTM). Eur J Cancer. 2017 Apr;75:284-98.

21 Capoluongo E, Ellison G, López-Guerrero JA, Penault-Llorca F, Ligtenberg MJ, Banerjee S, et al. Guidance Statement On BRCA1/2 Tumor Testing in Ovarian Cancer Patients. Semin Oncol. 2017 Jun;44(3):187-97.

22 Gori S, Barberis M, Bella MA, Buttitta F, Capoluongo E, Carrera P, et al.; AIOM-SIGU-SIBIOC-SIAPEC-IAP Working Group. Recommendations for the implementation of BRCA testing in ovarian cancer patients and their relatives. Crit Rev Oncol Hematol. 2019 Aug;140:67-72.

23 Pujol P, De La Motte Rouge T, Penault-Llorca F. From Targeting Somatic Mutations to Finding Inherited Cancer Predispositions: The Other Side of the Coin. Diagnostics (Basel). 2019 Jul 26;9(3):83.

24 Ludwig JA, Weinstein JN. Biomarkers in cancer staging, prognosis and treatment selection. Nat Rev Cancer. 2005 Nov;5(11):845-56. 
25 Eggener SE, Rumble RB, Armstrong AJ, Morgan TM, Crispino T, Cornford P, et al. Molecular Biomarkers in Localized Prostate Cancer: ASCO Guideline. J Clin Oncol. 2020 May;38(13):1474-94.

26 Taylor RA, Fraser M, Livingstone J, Espiritu SM, Thorne H, Huang V, et al. Germline BRCA2 mutations drive prostate cancers with distinct evolutionary trajectories. Nat Commun. 2017 Jan;8(1):13671.

27 Giri VN, Knudsen KE, Kelly WK, Cheng HH, Cooney KA, Cookson MS, et al. Implementation of Germline Testing for Prostate Cancer: Philadelphia Prostate Cancer Consensus Conference 2019 [published online ahead of print, 2020 Jun 9]. J Clin Oncol. 2020 Aug;38(24):2798-811.

28 Filella X, Foj L. Prostate Cancer Detection and Prognosis: From Prostate Specific Antigen (PSA) to Exosomal Biomarkers. Int J Mol Sci. 2016 Oct;17(11):1784.

29 Olmos D, Brewer D, Clark J, Danila DC, Parker C, Attard G, et al. Prognostic value of blood mRNA expression signatures in castration-resistant prostate cancer: a prospective, two-stage study. Lancet Oncol. 2012 Nov; 13(11):1114-24

30 Riely GL. What, When, and How of Biomarker Testing in Non-Small Cell Lung Cancer. J Natl Compr Canc Netw. 2017 May;15 5S:686-8.

31 Gregg JP, Li T, Yoneda KY. Molecular testing strategies in non-small cell lung cancer: optimizing the diagnostic journey. Transl Lung Cancer Res. 2019 Jun;8(3):286-301. [cited 2020 Jun 8] Available from: http://tlcr. amegroups.com/article/view/29030

32 Seijo LM, Peled N, Ajona D, Boeri M, Field JK, Sozzi G, et al. Biomarkers in Lung Cancer Screening: Achievements, Promises, and Challenges. J Thorac Oncol. 2019 Mar;14(3):343-57.

33 Campanella A, De Summa S, Tommasi S. Exhaled breath condensate biomarkers for lung cancer. J Breath Res. 2019 Aug;13(4):044002.

34 Field JK, Zulueta J, Veronesi G, Oudkerk M, Baldwin DR, Holst Pedersen J, et al. EU Policy on Lung Cancer CT Screening 2017. Biomed Hub. 2017 Nov;2 Suppl 1:154-61.

35 Duffy MJ, Sturgeon CM, Sölétormos G, Barak V, Molina R, Hayes DF, et al. Validation of new cancer biomarkers: a position statement from the European group on tumor markers. Clin Chem. 2015 Jun;61(6):809-20.

36 Kidess E, Heirich K, Wiggin M, Vysotskaia V, Visser BC, Marziali A, et al. Mutation profiling of tumor DNA from plasma and tumor tissue of colorectal cancer patients with a novel, high-sensitivity multiplexed mutation detection platform. Oncotarget. 2015 Feb;6(4):2549-61.

37 Filetti S, Durante C, Hartl D, Leboulleux S, Locati LD, Newbold K, et al.; ESMO Guidelines Committee. Electronic address: clinicalguidelines@esmo.org. Thyroid cancer: ESMO Clinical Practice Guidelines for diagnosis, treatment and follow-up†. Ann Oncol. 2019 Dec;30(12):1856-83.

38 Molecular testing for thyroid cancer can reduce unnecessary surgeries. Cited July 13, 2020. Available from: https://www.healio.com/news/endocrinology/20180309/molecular-testing-for-thyroid-cancer-canreduce-unnecessary-surgeries

39 Drilon A, Hu ZI, Lai GG, Tan DS. Targeting RET-driven cancers: lessons from evolving preclinical and clinical landscapes. Nat Rev Clin Oncol. 2018 Mar;15(3):151-67.

40 History of NTRKs in Cancer Biology. Cited July 13, 2020. Available from: http://ntrkfusions.com/history-ofntrks-in-cancer-biology/\#: :text=in \%20Cancer\%20Biology-,History\%20of\%20 NTRKs\%20in\%20 Cancer\%20Biology,was\%20discovered\%20in\%20the\%20colon.\&text=In\%201986\%2C\%20decades\%20 before $\% 20$ next,of\%20a\%20human $\% 20$ colon $\% 20$ carcinoma

41 Solit D, Rosen N. Oncogenic RAF: a brief history of time. Pigment Cell Melanoma Res. 2010 Dec;23(6):760-2.

42 Kamps, R., Brandão, R. D., Bosch, B. J., Paulussen, A. D., Xanthoulea, S., Blok, M. J., \& Romano, A. (2017). Nextgeneration sequencing in oncology: genetic

43 Tsimberidou AM, Hong DS, Ye Y, Cartwright C, Wheler JJ, Falchook GS, et al. Initiative for molecular profiling and advanced cancer therapy (IMPACT): an MD anderson precision medicine study. JCO Precis Oncol. 2017; 2017:10.1200/P0.17.00002.

44 Deverka PA, Douglas MP, Phillips KA. Use of Real-World Evidence in US Payer Coverage Decision-Making for Next-Generation Sequencing-Based Tests: Challenges, Opportunities, and Potential Solutions. Value Health. 2020 May;23(5):540-50.

45 Schneeweiss S. Real-World Evidence of Treatment Effects: The Useful and the Misleading. Clin Pharmacol Ther. 2019 Jul;106(1):43-4

46 Agarwala V, Khozin S, Singal G, O'Connell C, Kuk D, Li G, et al. Real-World Evidence In Support Of Precision Medicine: Clinico-Genomic Cancer Data As A Case Study. Health Aff (Millwood). 2018 May;37(5):765-72.

47 McKenzie AJ, H Dilks H, Jones SF, Burris H 3rd. Should next-generation sequencing tests be performed on all cancer patients? Expert Rev Mol Diagn. 2019 Feb;19(2):89-93.

48 Miksad RA, Samant MK, Sarkar S, Abernethy AP. Small But Mighty: The Use of Real-World Evidence to Inform Precision Medicine. Clin Pharmacol Ther. 2019 Jul;106(1):87-90.

49 Sherman RE, Anderson SA, Dal Pan GJ, Gray GW, Gross T, Hunter NL, et al. Real-world evidence - what is it and what can it tell us? N Engl J Med. 2016;375:2293e7.

50 ISO 20166-1:2018, Molecular in vitro diagnostic examinations - Specifications for pre-examination processes for formalin-fixed and paraffin-embedded (FFPE) tissue - Part 1: Isolated RNA. 2018 [accessed 29 June 2020]. https://www.iso.org/standard/67179.html

51 ISO 20166-2:2018, Molecular in vitro diagnostic examinations - Specifications for pre-examination processes for formalin-fixed and paraffin-embedded (FFPE) tissue - Part 2: Isolated proteins. 2018 [accessed 29 June 2020]. https://www.iso.org/standard/69802.html 
52 ISO 20166-3:2018, Molecular in vitro diagnostic examinations - Specifications for pre-examination processes for formalin-fixed and paraffin-embedded (FFPE) tissue - Part 3: Isolated DNA. 2018 [accessed 29 June 2020]. https://www.iso.org/standard/69803.html

53 ISO 20184-1:2018, Molecular in vitro diagnostic examinations - Specifications for pre-examination processes for frozen tissue - Part 1: Isolated RNA. 2018 [accessed 29 June 2020]. https://www.iso.org/standard/67215. html

54 ISO 20184-2:2018, Molecular in vitro diagnostic examinations - Specifications for pre-examination processes for frozen tissue - Part 2: Isolated proteins. 2018 [accessed 29 June 2020]. https://www.iso.org/ standard/69801.html

55 ISO 20186-1:2019, Molecular in vitro diagnostic examinations - Specifications for pre-examination processes for venous whole blood - Part 1: Isolated cellular RNA. 2019 [accessed 29 June 2020]. https://www.iso.org/ contents/data/standard/06/72/67217.html

56 ISO 20186-2:2019, Molecular in vitro diagnostic examinations - Specifications for pre-examination processes for venous whole blood - Part 2: Isolated genomic DNA. 2019 [accessed 29 June 2020]. https://www.iso.org/ contents/data/standard/06/97/69799.html

57 ISO 20186-3:2019, Molecular in vitro diagnostic examinations — Specifications for pre-examination processes for venous whole blood - Part 3: Isolated circulating cell free DNA from plasma. 2019 [accessed 29 June 2020]. https://www.iso.org/contents/data/standard/06/98/69800.html

58 CEN/TS 16826-3:2018, Molecular in vitro diagnostic examinations - Specifications for pre-examination processes for frozen tissue - Part 3: Isolated DNA. 2018 [accessed 29 June 2020]. https://standards.cen.eu/dyn/ www/f?p=204:110:0:FSP_PROJECT,FSP_ORG_ID:64590,6122\&cs=102897A2F2670733CDE2D41CA7921E5FF

59 CEN/TS 17305:2019, Molecular in vitro diagnostic examinations - Specifications for pre-examination processes for saliva - Isolated human DNA. 2019 [accessed 29 June 2020]. https://standards.cen.eu/dyn/ www/f?p=204:110:0:FSP_PROJECT:62239\&cs=130B991FE0957A5743F90225BBCACEABB

60 CEN/TS 17390-1:2020, Molecular in vitro diagnostic examinations - Specifications for pre-examination processes for circulating tumor cell (CTCs) in venous whole blood - Part 1: Isolated RNA. 2020 [accessed 29 June 2020]. https://standards.cen.eu/dyn/www/f?p=204:110:0:FSP_PROJECT:65450\&cs=1799CD1DD5E0 FBEEBE1246AC9CE6AB2F1

61 CEN/TS 17390-2:2020, Molecular in vitro diagnostic examinations - Specifications for pre-examination processes for circulating tumor cells (CTCs) in venous whole blood - Part 2: Isolated DNA. 2020 [accessed 29 June 2020]. https://standards.cen.eu/dyn/www/f?p=204:110:0:FSP_PROJECT:65452\&cs=1B8E3EC3BB49 D6FF8A6AA7684A31C1226

62 CEN/TS 17390-3:2020, Molecular in vitro diagnostic examinations - Specifications for pre-examination processes for circulating tumor cells (CTCs) in venous whole blood - Part 3: Preparations for analytical CTC staining. 2020 [accessed 29 June 2020]. https://standards.cen.eu/dyn/www/f?p=204:110:0:FSP_PROJECT: 65451\&cs=1406EA2E6FBA608D914F22B25BFD6A1BC

63 CEN/TS 16945, metabolomics in urine, serum and plasma. 2016 [accessed 29 June 2020]. https://standards. cen.eu/dyn/www/f?p=204:110:0:FSP_PROJECT:41043\&cs=172093B102C23FCCD97039A95FD18DA54

64 Annexes - Key Strategic Orientations. Annex 1 - Horizon Europe Cluster 1 Health. Cited June 8, 2020. Available from: https://ec.europa.eu/research/pdf/horizon-europe/annex-1.pdf

65 Chan TA, Yarchoan M, Jaffee E, Swanton C, Quezada SA, Stenzinger A, et al. Development of tumor mutation burden as an immunotherapy biomarker: utility for the oncology clinic. Ann Oncol. 2019 Jan;30(1):44-56.

66 National Academies of Sciences, Engineering, and Medicine; Health and Medicine Division; Board on Health Sciences Policy; Roundtable on Genomics and Precision Health. Understanding Disparities in Access to Genomic Medicine: Proceedings of a Workshop. Washington: National Academies Press; 2018 Nov 14. 2, Exploring the Barriers to Accessing Genomic and Genetic Services. Cited June 8, 2020. Available from: https:// www.ncbi.nlm.nih.gov/books/NBK538442/

67 Sturgeon C, Hill R, Hortin GL, Thompson D. Taking a new biomarker into routine use-a perspective from the routine clinical biochemistry laboratory. Proteomics Clin Appl. 2010 Dec;4(12):892-903.

68 Feinberg BA, Lang J, Grzegorczyk J, Stark D, Rybarczyk T, Leyden T, et al. Implementation of cancer clinical care pathways: a successful model of collaboration between payers and providers. J Oncol Pract. 2012 May; 8(3 Suppl):e38s-43s.

69 Ginsburg GS, Phillips KA. Precision medicine: from science to value. Health Aff (Millwood). 2018 May;37(5): 694-701.

70 Wurcel V, Perche O, Lesteven D, Williams DA, Schäfer B, Hopley C, et al. The value of companion diagnostics: overcoming access barriers to transform personalised health care into an affordable reality in Europe. Public Health Genomics. 2016;19(3):137-43.

71 Bogavac-Stanojevic N, Jelic-Ivanovic Z. The Cost-effective Laboratory: Implementation of Economic Evaluation of Laboratory Testing. J Med Biochem. 2017 Jul;36(3):238-42.

72 Wurcel V, Cicchetti A, Garrison L, Kip MMA, Koffijberg H, Kolbe A, et al. The Value of Diagnostic Information in Personalised Healthcare: A Comprehensive Concept to Facilitate Bringing This Technology into Healthcare Systems. Public Health Genomics. 2019;22(1-2):8-15.

73 Bieg-Bourne CC, Millis SZ, Piccioni DE, Fanta PT, Goldberg ME, Chmielecki J, et al. Next-generation sequencing in the clinical setting clarifies patient characteristics and potential actionability. Cancer Res. 2017 Nov;77(22): 6313-6320. https://doi.org/10.1158/0008-5472.CAN-17-1569. 
74 Garrison L, Mestre-Ferrandiz J, Zamora B. The value of knowing and knowing the value: improving the health technology assessment of complementary diagnostics. White paper. London: Office of Health Economics, EPEMED; 2016., Available at https://www.ohe.org/sites/default/files/WP_EpemedOHE_final.pdf

75 Enzmann H, Meyer R, Broich K. The new EU regulation on in vitro diagnostics: potential issues at the interface of medicines and companion diagnostics. Biomarkers Med. 2016 Dec;10(12):1261-8.

76 Colombage D, Lee S. Companion diagnostics: requirements under the new IVD regulation. Regulatory Rapporteur. 2018 Apr;15(4):18-23.

77 State of Health in the EU - Companion Report. European Union; 2019. ISBN: 978-92-76-10195-6. https://doi. org/10.2875/85326., EW-01-19-848-EN-N.

78 Zimmern RL. Testing challenges: evaluation of novel diagnostics and molecular biomarkers. Clin Med (Lond). 2009 Feb;9(1):68-73.

79 Drucker E, Krapfenbauer K. Pitfalls and limitations in translation from biomarker discovery to clinical utility in predictive and personalised medicine. EPMA J. 2013 Feb;4(1):7.

80 Mattiuzzi C, Lippi G. Which lessons shall we learn from the 2019 novel coronavirus outbreak? Ann Transl Med. 2020 Feb;8(3):48.

81 Council Recommendation of 2 December 2003 on Cancer screening (2003/878 EC). Cited June 8, 2020. Available from: https://ec.europa.eu/jrc/sites/jrcsh/files/2_December_2003\%20cancer\%20screening.pdf

82 HARMONY Project. Cited June 8, 2020. Available from: https://www.harmony-alliance.eu/en/about-us

83 Million European Genome initiative. Cited June 8, 2020. Available From: https://ec.europa.eu/digital-singlemarket/en/european-1-million-genomes-initiative

84 Médecine France génomique 2025. Cited June 8, 2020. Available from: https://solidarites-sante.gouv.fr/ systeme-de-sante-et-medico-social/recherche-et-innovation/france-genomique

85 EMA Regulatory Science to 2025 - Strategic reflection. EMA/110706/2020. Cited June 8, 2020. Available from: https://www.ema.europa.eu/en/documents/regulatory-procedural-guideline/ema-regulatory-science2025-strategic-reflection_en.pdf

86 Priestley P, Baber J, Lolkema MP, Steeghs N, de Bruijn E, Shale C, et al. Pan-cancer whole-genome analyses of metastatic solid tumours. Nature. 2019 Nov;575(7781):210-6.

87 Reitsma M, Fox J, Borre PV, Cavanaugh M, Chudnovsky Y, Erlich RL, et al. Effect of a Collaboration Between a Health Plan, Oncology Practice, and Comprehensive Genomic Profiling Company from the Payer Perspective. J Manag Care Spec Pharm. 2019 May;25(5):601-11.

88 Committee on Policy Issues in the Clinical Development and Use of Biomarkers for Molecularly Targeted Therapies; Board on Health Care Services. Institute of Medicine; National Academies of Sciences, Engineering, and Medicine; Graig LA, Phillips JK, Moses HL, editors. Supporting Data Infrastructure for Biomarker Tests for Molecularly Targeted Therapies. In: Biomarker Tests for Molecularly Targeted Therapies: Key to Unlocking Precision Medicine. Washington: National Academies Press; 2016. Available from: https://www.ncbi.nlm.nih. gov/books/NBK379334/

89 Morgan G, Aftimos P, Awada A. Current-day precision oncology: from cancer prevention, screening, drug development, and treatment - have we fallen short of the promise? Curr Opin Oncol. 2016;28:441e6.

90 Conley RB, Dickson D, Zenklusen JC, Al Naber J, Messner DA, Atasoy A, et al. Core clinical data elements for cancergenomic repositories: a multi-stakeholder consensus. Cell.2017;171:982e6.https://doi.org/10.1016/j. cell.2017.10.032.

91 Morash M, Mitchell H, Beltran H, Elemento O, Pathak J. The role of next-generation sequencing in precision medicine: a review of outcomes in oncology. J Pers Med. 2018 Sep 17;8(3):30.

92 Horgan D, Bernini C, Thomas PP, Morre SA. Cooperating on Data: The Missing Element in Bringing Real Innovation to Europe's Healthcare Systems. Public Health Genomics. 2019;22(3-4):77-101.

93 Ehmann F, Caneva L, Prasad K, Paulmichl M, Maliepaard M, Llerena A, et al. Pharmacogenomic information in drug labels: European Medicines Agency perspective. Pharmacogenomics J. 2015 Jun;15(3):201-10.

94 Agarwala V, Khozin S, Singal G, O'Connell C, Kuk D, Li G, et al. Real-world evidence in support of precision medicine: clinico-genomic cancer data as a case study. Health Aff. 2018 May;37(5):765-772. https://doi. org/10.1377/hlthaff.2017.1579.

95 Cutler DM. Early Returns From the Era of Precision Medicine. JAMA. 2020 Jan;323(2):109-10.

96 Krieckaert CL, Nair SC, Nurmohamed MT, van Dongen CJ, Lems WF, Lafeber FP, et al. Personalised treatment using serum drug levels of adalimumab in patients with rheumatoid arthritis: an evaluation of costs and effects. Ann Rheum Dis. 2015 Feb;74(2):361-8.

97 Horvath AR, Lord SJ, StJohn A, Sandberg S, Cobbaert CM, Lorenz S, et al.; Test Evaluation Working Group of the European Federation of Clinical Chemistry Laboratory Medicine. From biomarkers to medical tests: the changing landscape of test evaluation. Clin Chim Acta. 2014 Jan;427:49-57.

98 Gray SW, Hicks-Courant K, Cronin A, Rollins BJ, Weeks JC. Physicians' attitudes about multiplex tumor genomic testing. J Clin Oncol. 2014 May;32(13):1317-23.

99 Horgan D, Jansen M, Leyens L, Lal JA, Sundbrak R, Hackenitz E, et al. An index of barriers for the implementation of personalised medicine and pharmacogenomics in Europe. Public Health Genomics. 2014;17(5-6): 287-98. 